\title{
Large Eddy Simulation of a spray jet flame using Doubly Conditional Moment Closure
}

\author{
M. P. Sitte*, E. Mastorakos \\ University of Cambridge, Department of Engineering, CB2 PZ1, Cambridge, UK.
}

\begin{abstract}
A spray jet flame is modelled using Large Eddy Simulation (LES) with Doubly Conditional Moment Closure (DCMC). Since turbulent spray flames may include multiple combustion modes, the DCMC model uses both mixture fraction and reaction progress variable as conditioning variables. Conditional spray terms were included in the DCMC model to consider the coupling between evaporation and the flame structure. In the case of spatial homogeneity and in the limit of negligible mixture fraction scalar dissipation rate (SDR), the DCMC equation is shown to reproduce the flame structure of freely propagating laminar flames. For the spray jet flame, a good agreement between the simulation results and the experiments is achieved, in terms of the spray statistics, as well as the instantaneous and mean flame shape. The simulation shows important differences in the flame structure between the turbulent inner and the quasilaminar outer flame branch. The doubly-conditional parametrisation appears to be advantageous for resolving small scale effects related to droplet evaporation. Analysis of the DCMC equation suggests that the behaviour of the flame at its anchoring point is strongly influenced by non-premixed burning modes. The solution appears to be weakly affected by terms of convective transport in the DCMC equation, but significant spatial variations and temporal fluctuations of the conditional reaction rate, around $10 \%$ of the time-based mean, persist.
\end{abstract} Keywords: Large Eddy Simulation, DCMC, mixture fraction, reaction

*Corresponding author. mps59@cam.ac.uk 
progress variable, spray combustion, n-heptane

\section{Introduction}

Spray combustion is relevant to a large number of technical applications but it is very challenging from a modelling perspective, even under the assumption of a dilute spray. This is due to the wide range of length and time scales involved

5 and the strong coupling of turbulence, evaporation and chemical reaction [1, 2], which manifests in complex combustion regimes [3] and a diverse palette of macroscopic phenomena, including flame propagation 4, ignition characteristics [5] and extinction [6], which differ from common experience with gaseous flames.

Therefore, in order to reproduce the behaviour of a spray flame in a numerical simulation, an advanced combustion model is required. The modelling approach should include the interactions of both evaporation and micro-mixing with the flame structure. Moreover, online solution of the chemistry is necessary to account for transient effects on the flame structure. This can be achieved using Large Eddy Simulation (LES) and the Conditional Moment Closure (CMC) framework for turbulent combustion modelling. CMC modelling is well-established (see reviews by Klimenko and Bilger [7 and Kronenburg and Mastorakos [8]) and LES-CMC [9] particularly has so far been successfully applied to various combustion problems, including forced ignition [10, the stabilisation of lifted non-premixed jet flames [11, local extinction and blow-off in non-premixed [12, 13, and spray flames [14, and the behaviour of premixed flames approaching blow-off [15. In all these cases a single conditioning variable was used to parametrise the flame structure; either the mixture fraction or the reaction progress variable in the rare cases where CMC was applied to a premixed flame. While the statistical foundation of CMC ensures that it is, in principle, not limited to a specific combustion mode, in practice, CMC closure for the chemical reaction source term requires the flame to be reasonably well parametrised by the conditioning variable [7. Consequently, conventional 
singly-conditioned CMC models are limited to cases that are either premixed or predominantly non-premixed, which is also the case of the above-mentioned spray flames that were simulated using CMC. In contrast, a general type of spray flame can span a broad range of combustion modes from non-premixed to almost fully pre-vaporised and premixed. These modes may also co-exist within a single flame [16]. In particular, turbulent spray flames with increasing degrees of premixed behaviour have recently received more attention from experimental [17, 18] and numerical points of view [19, 20. This reflects modern design trends - for example, in aero-engine combustors, which operate in partially premixed conditions in order to reduce pollutant formation and improve combustion efficiency.

40 This motivates the development of Doubly Conditional Moment Closure (DCMC) where two conditioning variables provide a sufficient parameter space for the flame structure. To date, DCMC has primarily been applied at the level of fundamental feasibility studies, where DCMC was tested against a Direct Numerical Simulation (DNS) and closure for the DCMC equation was provided from DNS results. These studies focused on predicting extinction [21, 22, 23, and the effect of temperature inhomogeneity on ignition [24] and have so far demonstrated a great potential of the modelling approach for predicting complex, transient combustion phenomena. To the knowledge of the authors, the only simulation of a lab-scale flame using DCMC, coupled with a Reynolds-Averaged Navier Stokes (RANS) computation, has been performed by Sitte and Mastorakos [25]. From a broader perspective, the strategy of double-conditioning has also been recently employed in the CMC-related modelling approach of Conditional Source-term Estimation (CSE) 26].

In this work, we present an application of the LES-DCMC approach, based 55 on mixture fraction and reaction progress variable as conditioning variables. This allows parametrisation of the whole range from non-premixed to fully premixed flames. Moreover, the effect of liquid droplet evaporation on the flame structure is considered by modelling the spray terms in conditional space. Inclusion of conditional evaporation terms in the model equation is challenging and 
has so far only been attempted for singly-conditional CMC in a small number of publications [14, 27, 28, 29, 30, 31, 32. A model for the doubly-conditional spray terms has previously been proposed but was not included in the DCMC model equation [25].

The LES-DCMC model is employed to simulate a spray jet flame recently studied experimentally [33, 34, 35]. This test flame has already been simulated using: LES with the Thickened Flame Model in the limit of a resolved flame [33, LES with the Stochastic Fields Method [36], LES with Filtered Tabulated Chemistry [37] and RANS with Flamelet Generated Manifolds 38.

The article is organised as follows. First, the modelling approach for LESDCMC and the spray is detailed. Second, general features of a solution of the DCMC equation are discussed for the simplified case of spatial homogeneity, denoted as "DCMC-0D". Third, comparisons between simulation results and experimental data for the test flame investigated are shown for validation of the present modelling approach and interesting features of the flame are discussed.

75 Fourth, the DCMC modelling approach is discussed by analysing spatial and temporal evolution of the flame structure parametrised in doubly-conditional space. At the end of the article conclusions and recommendations for further work are given.

\section{Methodology}

\subsection{General approach}

The numerical simulation follows an Euler-Lagrangian approach, where the flow field of the continuous gas phase was computed by a Large Eddy Simulation (LES). A dilute spray was assumed, such that the effect of liquid volume fraction on the LES-filtered conservation equations can be neglected. The dis-

85 persed liquid phase was modelled as Lagrangian particles that represent parcels of liquid droplets. The Doubly Conditional Moment Closure (DCMC) model is used to solve for the spatial and temporal evolution of the local flame structure, 
which is parametrised based in two conditioning variables, mixture fraction $\xi$ and reaction progress variable $c$.

The mixture fraction acts as a passive scalar with respect to chemical reaction, zero denoting pure air and one pure fuel vapour. The reaction progress variable is defined as a linear function of a reactive scalar $Y_{\psi}$, where zero represents the mixing line and one the equilibrium mass fraction, as introduced by Bray et al. 39 .

$$
c(\mathbf{x}, t)=c_{\psi}\left(\xi(\mathbf{x}, t), Y_{\psi}(\mathbf{x}, t)\right)=\frac{Y_{\psi}^{0}(\xi(\mathbf{x}, t))-Y_{\psi}(\mathbf{x}, t)}{Y_{\psi}^{0}(\xi(\mathbf{x}, t))-Y_{\psi}^{\mathrm{Eq}}(\xi(\mathbf{x}, t))}
$$

In the present work $c$ is based on the mass fraction of carbon dioxide, $Y_{\psi}=Y_{\mathrm{CO} 2}$.

\subsection{Continuous phase}

For the gas phase, the continuity equation, momentum equation and transport equations for the LES-filtered values and sub-grid scale (sgs) variances of the conditioning variables, and the filtered enthalpy are solved. The LES-filtered equations for continuity and momentum are

$$
\begin{gathered}
\frac{\partial \bar{\rho}}{\partial t}+\nabla \cdot(\bar{\rho} \tilde{\mathbf{u}})=\bar{\rho} \widetilde{\Pi} \\
\frac{\partial \bar{\rho} \tilde{\mathbf{u}}}{\partial t}+\nabla \cdot(\bar{\rho} \tilde{\mathbf{u}} \tilde{\mathbf{u}})=-\nabla \bar{p}+\nabla \cdot \overline{\boldsymbol{\tau}}+\nabla \cdot \overline{\boldsymbol{\tau}}_{\mathrm{sgs}}+\overline{\mathbf{S}}_{u}
\end{gathered}
$$

where $\overline{\boldsymbol{\tau}}$ is the viscous stress tensor and $\overline{\boldsymbol{\tau}}_{\text {sgs }}$ is the turbulent sgs stress tensor, which is closed with a constant Smagorinsky model. The sgs viscosity is computed as $\mu_{\mathrm{sgs}}=c_{k} \bar{\rho} \Delta k_{\mathrm{sgs}}$, where $\Delta$ is the filter width equal to the cubic root of an LES cell, $k_{\mathrm{sgs}}$ is the sgs kinetic energy and $c_{k}=0.02$ is a model constant [40]. $\widetilde{I}$ is the volumetric rate of phase change per unit volume and $\overline{\mathbf{S}}_{u}$ represents the transfer of momentum from the liquid to the gas phase.

The transport equations for the LES-filtered values and the sgs variances of the conditioning variables, mixture fraction and reaction progress variable, are

$$
\frac{\partial \bar{\rho} \tilde{\xi}}{\partial t}+\nabla \cdot(\bar{\rho} \tilde{\mathbf{u}} \tilde{\xi})=\nabla \cdot\left(\bar{\rho}\left(D_{t}+\bar{D}\right) \nabla \tilde{\xi}\right)+\bar{\rho} \widetilde{\Pi}
$$




$$
\begin{gathered}
\frac{\partial \bar{\rho} \widetilde{\xi^{\prime \prime 2}}}{\partial t}+\nabla \cdot\left(\bar{\rho} \tilde{\mathbf{u}} \widetilde{\xi^{\prime \prime 2}}\right)=\nabla \cdot\left(\bar{\rho}\left(D_{t}+\bar{D}\right) \nabla \widetilde{\xi^{\prime \prime 2}}\right)-2 \bar{\rho} \widetilde{N}_{\xi} \\
+2 \bar{\rho}\left(D_{t}+\bar{D}\right) \nabla \tilde{\xi} \cdot \nabla \tilde{\xi} \\
\frac{\partial \bar{\rho} \tilde{c}}{\partial t}+\nabla \cdot(\bar{\rho} \tilde{\mathbf{u}} \tilde{c})=\nabla \cdot\left(\bar{\rho}\left(D_{t}+\bar{D}\right) \nabla \tilde{c}\right)+\bar{\rho} \widetilde{\dot{\omega}_{c}^{*}}+\bar{\rho} \tilde{c} \widetilde{\Pi} \\
\frac{\partial \bar{\rho}{c^{\prime \prime 2}}^{\partial t}+\nabla \cdot\left(\bar{\rho} \tilde{\mathbf{u}} \widetilde{c^{\prime \prime 2}}\right)=}{}+\left(\bar{\rho}\left(D_{t}+\bar{D}\right) \nabla \widetilde{c^{\prime \prime 2}}\right)-2 \bar{\rho} \widetilde{N_{c}} \\
+2 \bar{\rho}\left(D_{t}+\bar{D}\right) \nabla \tilde{c} \cdot \nabla \tilde{c}+2 \bar{\rho}\left(\widetilde{c \dot{\omega}_{c}^{*}}-\tilde{c} \widetilde{\dot{\omega}_{c}^{*}}\right)
\end{gathered}
$$

where the molecular and turbulent diffusivities are computed as $\bar{D}=\bar{\mu} /(\bar{\rho} \mathrm{Sc})$ and $D_{\mathrm{t}}=\mu_{\mathrm{sgs}} /\left(\bar{\rho} \mathrm{Sc}_{t}\right)$ with $\mathrm{Sc}=0.7$ and $\mathrm{Sc}_{t}=0.4$ respectively. The scalar dissipation rates (SDRs) are composed of a resolved part and a sub-grid scale contribution. Hence, the SDR of the mixture fraction is computed as

$$
\widetilde{N}_{\xi}=\underbrace{\bar{D} \nabla \tilde{\xi} \cdot \nabla \tilde{\xi}}_{\widetilde{N}_{\xi, \text { res }}}+\underbrace{\frac{1}{2} C_{N} \frac{\mu_{\text {sgs }}}{\Delta^{2}} \widetilde{\xi^{\prime \prime 2}}}_{\widetilde{N}_{\xi, \text { sgs }}}
$$

where $C_{N}=42$ 41]. In the same way, the SDR of the reaction progress variable is

$$
\widetilde{N}_{c}=\bar{D} \nabla \tilde{c} \cdot \nabla \tilde{c}+\widetilde{N}_{c, \mathrm{sgs}}
$$

where the sub-grid scale contribution is closed using an algebraic model that includes flame dilatation effects [42, in the version adapted for stratified mixture 43 .

$$
\widetilde{N}_{c, \mathrm{sgs}}=\left(1-e^{-0.75 \Delta / \delta_{L}^{0}}\right)\left[2 K_{c} \frac{S_{L}^{0}}{\delta_{L}^{0}}+\left(C_{3}-\tau C_{4} \mathrm{Da}_{\mathrm{sgs}}\right) \frac{2 u_{\mathrm{sgs}}^{\prime}}{3 \Delta}\right] \frac{\widetilde{c^{\prime \prime 2}}}{\beta_{c}}
$$

where $S_{L}^{0}$ is the laminar flame speed, $\delta_{L}^{0}$ is the thermal flame thickness, $\tau$ is the normalised temperature increase and $\mathrm{Da}_{\mathrm{sgs}}$ is the sgs Darmköhler number. $S_{L}^{0}$, $\delta_{L}^{0}$ and $\tau$ are calculated as functions of $\tilde{\xi}$ [4]. The sgs velocity fluctuation $u_{\mathrm{sgs}}^{\prime}$ is computed based on the scaled-similarity hypothesis [44] using a test filter of size $2 \Delta$. This procedure was used with the SDR model in Refs [43, 45]. The model 
parameters, $K_{c}, C_{3}$ and $C_{4}$, are computed as in Ref. [42, and, in particular, a constant value $\beta_{c}=7.5$ was used [43]. The progress variable equation contains the source term $\widetilde{\dot{\omega}_{c}^{*}}$, which is the filtered value of the apparent reaction rate, as first derived for a scaled progress variable by Bray et al. 39.

$$
\dot{\omega}_{c}^{*}=\frac{1}{\partial Y_{\psi} / \partial c}\left[\dot{\omega}_{\psi}+N_{\xi} \frac{\partial^{2} Y_{\psi}}{\partial \xi^{2}}+2 N_{\xi c} \frac{\partial^{2} Y_{\psi}}{\partial \xi \partial c}+N_{c} \frac{\partial^{2} Y_{\psi}}{\partial c^{2}}\right]
$$

Since $c$ depends linearly on $Y_{\psi}$ and $N_{\xi c}=0$ is assumed (see modelling in section 2.4, the apparent reaction rate $\dot{\omega}_{c}^{*}$ consists of two contributions, $\dot{\omega}_{c}$ due to chemical reaction and $\dot{\omega}_{p}$ due to passive scalar mixing. Then LES-filtered value is

$$
\widetilde{\rho} \widetilde{\dot{\omega}_{c}^{*}}=\widetilde{\rho} \widetilde{\dot{\omega}_{c}}+\widetilde{\rho} \widetilde{\dot{\omega}_{p}}=\overline{\frac{1}{\partial Y_{\psi} / \partial c} \rho \dot{\omega}_{\psi}}+\overline{\frac{1}{\partial Y_{\psi} / \partial c} \rho N_{\xi} \frac{\partial^{2} Y_{\psi}}{\partial \xi^{2}}}
$$

Closure for $\widetilde{\dot{\omega}_{c}^{*}}$ and $\widetilde{c \dot{\omega}_{c}^{*}}$ is provided by the DCMC model, detailed later. The derivation of transport equations for the filtered values and the sgs variances of the mixture fraction and the scaled reaction progress variable leads to spray source terms in each equation [25]. The spray source term in Eqn. 4 is exact and the following modelling choices were made for the other transport equations: the evaporation source in the $\widetilde{\xi^{\prime \prime 2}}$-equation is not considered in the present work, since currently available sub-models for $\widetilde{N}_{\xi}$ seem unsuitable to balance the production of variance in regions with low turbulence level - evaporation in regions with lower turbulence intensity constitutes a main difference between the present case and the flame studied by Giusti and Mastorakos [14] who successfully included this source term. The effect of evaporation on $\widetilde{c^{\prime \prime 2}}$ was neglected and the source to the $\tilde{c}$-equation was modelled as discussed by Sitte and Mastorakos [25].

Evaporative cooling very directly affects the temperature in the vicinity of droplets, and even small temperature differences can have a strong effect on the evaporation rate. Therefore, the effect needs to be considered locally at small scale, which makes it difficult to consider even with a DCMC approach, since solving the $Q_{h}$ equation intrinsically involves an averaging procedure. For this reason a transport equation for the LES-filtered enthalpy is solved

$$
\frac{\partial \bar{\rho} \tilde{h}}{\partial t}+\nabla \cdot(\bar{\rho} \tilde{h} \widetilde{\mathbf{u}})=\nabla \cdot\left(\bar{\rho}\left(a_{t}+\bar{a}\right) \nabla \tilde{h}\right)+\bar{\rho} \tilde{S}_{h}
$$


following. The filtered values $\bar{\rho}_{\mathrm{cmc}}, \tilde{T}_{\mathrm{cmc}}, \bar{C}_{p, \mathrm{cmc}}$ and $\tilde{h}_{\mathrm{cmc}}$ are computed by integrating the corresponding conditional variables with the filtered probability density function (FDF). The filtered values, $\bar{\rho}$ and $\tilde{T}$, are obtained by performing a linear correction, $\tilde{T}=\tilde{T}_{\mathrm{cmc}}+\left(\tilde{h}-\tilde{h}_{\mathrm{cmc}}\right) / \bar{C}_{p, \mathrm{cmc}}$ and $\bar{\rho}=\bar{\rho}_{\mathrm{cmc}}\left(\tilde{T}_{\mathrm{cmc}} / \tilde{T}\right)$. The is small. The enthalpy equation contains a spray source term $\bar{\rho} \tilde{S}_{h}$, which is computed for each Lagrangian parcel by the evaporation model discussed later. Following a unity Lewis number assumption, in the present work, molecular and turbulent thermal diffusivities, $\bar{a}$ and $a_{t}$, are identical with the respective mass

\subsection{Dispersed phase}

For the dispersed phase equations for position, momentum, mass and temperature are solved considering two-way coupling with the continuous phase. No model for secondary break-up was used. The droplets experience sphere drag computed according to the Schiller-Naumann correlation [46]. Gravitational acceleration and stochastic dispersion were neglected for large Froude number and low sgs kinetic energy respectively. Evaporation is given by the Abramzon and Sirignano model [4] with Stefan flow correction, non-unity Lewis number in the film and the assumption of infinite conductivity for the liquid. The LES

Additional details can be found in the "Supplementary Material".

\subsection{Doubly Conditional Moment Closure}

In the context of LES, the CMC model is based on a conditional filtering procedure of the local instantaneous transport equations [9]. In the present DCMC approach the conditional moment of a reactive scalar $Y$ is written as

$$
Q(\eta, \zeta ; \mathbf{x}, t)=\langle Y(\mathbf{x}, t) \mid \xi(\mathbf{x}, t)=\eta, c(\mathbf{x}, t)=\zeta\rangle
$$

where $\eta$ and $\zeta$ are the sample space variables of mixture fraction and reaction progress variable respectively. 
The DCMC model equation for spray flames was presented by Sitte and Mastorakos [25]. The conditional spray terms were derived using the approach by Mortensen and Bilger [48. The transport equation for the conditional moments of species mass fractions $Q_{\alpha}=\left\langle Y_{\alpha} \mid \eta, \zeta\right\rangle$ can be written as follows,

$$
\begin{aligned}
\frac{\partial Q_{\alpha}}{\partial t} & +\nabla \cdot\left(Q_{\alpha}\langle\mathbf{u} \mid \eta, \zeta\rangle\right)=Q_{\alpha} \nabla \cdot(\langle\mathbf{u} \mid \eta, \zeta\rangle) \\
& -\frac{1}{\bar{\rho} \widetilde{p}} \nabla \cdot\left(\bar{\rho} \widetilde{p}\left[\left\langle\mathbf{u} Y_{\alpha} \mid \eta, \zeta\right\rangle-Q_{\alpha}\langle\mathbf{u} \mid \eta, \zeta\rangle\right]\right)+\frac{1}{\bar{\rho} \widetilde{p}} \nabla \cdot\left(\bar{\rho} \widetilde{p}\left\langle D \nabla Y_{\alpha} \mid \eta, \zeta\right\rangle\right) \\
& +\left\langle N_{\xi} \mid \eta, \zeta\right\rangle \frac{\partial^{2} Q_{\alpha}}{\partial \eta^{2}}+2\left\langle N_{\xi c} \mid \eta, \zeta\right\rangle \frac{\partial^{2} Q_{\alpha}}{\partial \eta \partial \zeta}+\left\langle N_{c} \mid \eta, \zeta\right\rangle \frac{\partial^{2} Q_{\alpha}}{\partial \zeta^{2}} \\
& +\left\langle\dot{\omega}_{\alpha} \mid \eta, \zeta\right\rangle-\left\langle\dot{\omega}_{c}^{*} \mid \eta, \zeta\right\rangle \frac{\partial Q_{\alpha}}{\partial \zeta} \\
& +\left(\delta_{\alpha \mathrm{F}}-Q_{\alpha}\right)\langle\Pi \mid \eta, \zeta\rangle-\left\langle S_{\xi}^{-} \mid \eta, \zeta\right\rangle \frac{\partial Q_{\alpha}}{\partial \eta}-\left\langle S_{c}^{-} \mid \eta, \zeta\right\rangle \frac{\partial Q_{\alpha}}{\partial \zeta}
\end{aligned}
$$

where

$$
\begin{gathered}
\left\langle\dot{\omega}_{c}^{*} \mid \eta, \zeta\right\rangle=\frac{1}{\partial Q_{\psi} / \partial \zeta} \\
\times\left[\left\langle\dot{\omega}_{\psi} \mid \eta, \zeta\right\rangle+\left\langle N_{\xi} \mid \eta, \zeta\right\rangle \frac{\partial^{2} Q_{\psi}}{\partial \eta^{2}}+2\left\langle N_{\xi c} \mid \eta, \zeta\right\rangle \frac{\partial^{2} Q_{\psi}}{\partial \eta \partial \zeta}+\left\langle N_{c} \mid \eta, \zeta\right\rangle \frac{\partial^{2} Q_{\psi}}{\partial \zeta^{2}}\right] \\
\left\langle S_{\xi}^{-} \mid \eta, \zeta\right\rangle=(1-\eta)\langle\Pi \mid \eta, \zeta\rangle \\
\left\langle S_{c}^{-} \mid \eta, \zeta\right\rangle=\frac{1}{\partial Q_{\psi} / \partial \zeta}\left[\delta_{\psi \mathrm{F}}-Q_{\psi}-(1-\eta) \frac{\partial Q_{\psi}}{\partial \eta}\right]\langle\Pi \mid \eta, \zeta\rangle
\end{gathered}
$$

Here $\langle\cdot \mid \eta, \zeta\rangle$ represents density-weighted conditional filtering, $\widetilde{p}=\widetilde{p}(\eta, \zeta)$ is the density-weighted FDF and $\bar{\rho}$ is the unconditionally filtered density. The term $\left\langle\dot{\omega}_{c}^{*} \mid \eta, \zeta\right\rangle$ represents the conditionally averaged apparent reaction rate in the $c$ equation; $\left\langle S_{\xi}^{-} \mid \eta, \zeta\right\rangle$ and $\left\langle S_{c}^{-} \mid \eta, \zeta\right\rangle$ are the conditionally averaged spray source terms of the mixture fraction and reaction progress variable equations in nonconservative formulation respectively (see "Supplementary Material"). Here we used the Kronecker delta, $\delta_{\alpha \mathrm{F}}=1$ if $\alpha$ is the fuel species, zero otherwise; and similar for $\delta_{\psi \mathrm{F}}$. The transport in physical space was re-arranged as an advective and a dilatation term, as in Refs [49, 12. In addition to turbulent transport, the molecular diffusion in physical space was retained, since both terms can be 
in certain cases it might be appropriate to relax this condition and, for instance, let the boundary at $\zeta=1$ evolve in time. For completeness, the changes to the DCMC equation, that occur in that case, can be found in the "Supplementary Material".

Similar to the DCMC equation for species mass fraction (Eqn. 15), the transport equation for the doubly-conditional enthalpy $Q_{h}=\langle h \mid \eta, \zeta\rangle$ is

$$
\begin{aligned}
\frac{\partial Q_{h}}{\partial t} & +\nabla \cdot\left(Q_{h}\langle\mathbf{u} \mid \eta, \zeta\rangle\right)=Q_{h} \nabla \cdot(\langle\mathbf{u} \mid \eta, \zeta\rangle) \\
& -\frac{1}{\bar{\rho} \widetilde{p}} \nabla \cdot\left(\bar{\rho} \widetilde{p}\left[\langle\mathbf{u} h \mid \eta, \zeta\rangle-Q_{h}\langle\mathbf{u} \mid \eta, \zeta\rangle\right]\right)+\frac{1}{\bar{\rho} \widetilde{p}} \nabla \cdot(\bar{\rho} \widetilde{p}\langle a \nabla h \mid \eta, \zeta\rangle) \\
& +\left\langle N_{\xi} \mid \eta, \zeta\right\rangle \frac{\partial^{2} Q_{h}}{\partial \eta^{2}}+2\left\langle N_{\xi c} \mid \eta, \zeta\right\rangle \frac{\partial^{2} Q_{h}}{\partial \eta \partial \zeta}+\left\langle N_{c} \mid \eta, \zeta\right\rangle \frac{\partial^{2} Q_{h}}{\partial \zeta^{2}} \\
& -\left\langle\dot{\omega}_{c}^{*} \mid \eta, \zeta\right\rangle \frac{\partial Q_{h}}{\partial \zeta} \\
& +\left\langle S_{h} \mid \eta, \zeta\right\rangle-Q_{h}\langle\Pi \mid \eta, \zeta\rangle-\left\langle S_{\xi}^{-} \mid \eta, \zeta\right\rangle \frac{\partial Q_{h}}{\partial \eta}-\left\langle S_{c}^{-} \mid \eta, \zeta\right\rangle \frac{\partial Q_{h}}{\partial \zeta}
\end{aligned}
$$

where $a$ is the thermal diffusivity equal to $D$ for unity Lewis number. $\left\langle S_{h} \mid \eta, \zeta\right\rangle$ is the conditional enthalpy source due to evaporation.

The DCMC equation is discretised using a finite volume formulation as detailed in Refs [12, 49], where CMC is solved on a grid that is significantly coarser than then LES resolution. Unconditionally filtered quantities are computed by integrating their conditional moments with the FDF. The FDF is presumed as the product of two $\beta$-PDFs, $\tilde{p}(\eta, \zeta)=p_{\beta}\left(\eta ; \tilde{\xi}, \widetilde{\xi^{\prime \prime 2}}\right) \times p_{\beta}\left(\zeta ; \tilde{c}, \widetilde{c^{\prime \prime 2}}\right)$, hence, not considering the cross-correlation of $\xi$ and $c$.

For the unclosed terms in the DCMC equation models need to be provided. First order closure is applied to the conditional reaction rate $\left\langle\dot{\omega}_{\alpha} \mid \eta, \zeta\right\rangle$. The conditional velocity is modelled as $\langle\mathbf{u} \mid \eta, \zeta\rangle=\tilde{\mathbf{u}}$. The molecular diffusion term 
was closed as, $\left\langle D \nabla Y_{\alpha} \mid \eta, \zeta\right\rangle=\bar{D} \nabla Q_{\alpha}$, and for the turbulent transport the gradient diffusion assumption is employed, $\left[\left\langle\mathbf{u} Y_{\alpha} \mid \eta, \zeta\right\rangle-Q_{\alpha}\langle\mathbf{u} \mid \eta, \zeta\rangle\right]=-D_{t} \nabla Q_{\alpha}$. Following the unstructured implementation [12, 49], the conditional SDRs at the $\mathrm{CMC}$ resolution are obtained by presuming their shape at the level of the LES cells and then conditionally volume averaging over the whole CMC,

$$
\langle N \mid \eta, \zeta\rangle_{\mathrm{cmc}}=\frac{\int_{V_{\mathrm{cmc}}} \bar{\rho} \tilde{p}(\eta, \zeta)\langle N \mid \eta, \zeta\rangle_{\text {les }} \mathrm{d} V}{\int_{V_{\mathrm{cmc}}} \bar{\rho} \tilde{p}(\eta, \zeta) \mathrm{d} V}
$$

For the presumed conditional SDRs at the LES resolution, the following models are used. It is assumed that, $\left\langle N_{\xi} \mid \eta, \zeta\right\rangle \simeq\left\langle N_{\xi} \mid \eta\right\rangle$ [50] and the Amplitude Mapping Closure (AMC) model [51] is used, $\left\langle N_{\xi} \mid \eta, \zeta\right\rangle_{\text {les }}=N_{0} G(\eta)$. Here the bell curve $G(\eta)=\exp \left(-2\left[\operatorname{erf}^{-1}(2 \eta-1)\right]^{2}\right)$ is the presumed shape of the SDR, which is scaled so that integrating with the FDF gives the filtered value, viz. $N_{0}=$ $\tilde{N}_{\xi} / \int_{0}^{1} \int_{0}^{1} G(\eta) \tilde{p}(\eta, \zeta) \mathrm{d} \eta \mathrm{d} \zeta$. The same principle is employed for $\left\langle N_{c} \mid \eta, \zeta\right\rangle_{\text {les }}$, using a tabulation the SDR from freely propagating laminar premixed flames $N_{c}^{0}(\eta, \zeta)$ as the presumed shape (see Fig. 2 and scaling according to $\widetilde{N}_{c}$. The cross-scalar dissipation rate is modelled as $\left\langle N_{\xi c} \mid \eta, \zeta\right\rangle_{\text {les }}=0$, which is consistent with the presumed shape of the FDF assuming statistical independence of the conditioning variables.

The conditional apparent reaction rate is closed according to Eqn. 16. Since $\partial^{2} Q_{\psi} / \partial \zeta^{2}=0$ and $\left\langle N_{\xi c} \mid \eta, \zeta\right\rangle=0$ two finite terms remain, the first one is due to chemical reaction, the second due to passive scalar mixing.

$$
\left\langle\dot{\omega}_{c}^{*} \mid \eta, \zeta\right\rangle=\underbrace{\frac{1}{\partial Q_{\psi} / \partial \zeta}\left\langle\dot{\omega}_{\psi} \mid \eta, \zeta\right\rangle}_{\left\langle\dot{\omega}_{c} \mid \eta, \zeta\right\rangle}+\underbrace{\frac{1}{\partial Q_{\psi} / \partial \zeta}\left\langle N_{\xi} \mid \eta, \zeta\right\rangle \frac{\partial^{2} Q_{\psi}}{\partial \eta^{2}}}_{\left\langle\dot{\omega}_{p} \mid \eta, \zeta\right\rangle}
$$

Closure for the LES-filtered apparent reaction rate $\widetilde{\dot{\omega}_{c}^{*}}$ is provided by integrating $175\left\langle\dot{\omega}_{c}^{*} \mid \eta, \zeta\right\rangle$ with the FDF, using the locally presumed conditional SDR $\left\langle N_{\xi} \mid \eta, \zeta\right\rangle_{\text {les }}$ to compute $\left\langle\dot{\omega}_{p} \mid \eta, \zeta\right\rangle$. Simmilarly, $\widetilde{c \dot{\omega}_{c}^{*}}$ is computed by integrating $\zeta\left\langle\dot{\omega}_{c}^{*} \mid \eta, \zeta\right\rangle$ with the FDF.

The conditional volumetric evaporation rate $\langle\Pi \mid \eta, \zeta\rangle_{\text {les }}$ is modelled as a ridge in $(\eta, \zeta)$-space along the iso-contour given by $\left[Q_{\mathrm{F}}(\eta, \zeta)=\left\langle Y_{\mathrm{F} s}\right\rangle_{\text {les }}\right][25$, where $Q_{\mathrm{F}}$ is the conditional mean of fuel mass fraction and $\left\langle Y_{\mathrm{F} s}\right\rangle_{\text {les }}$ is the fuel mass 
fraction on the droplet surface computed from the vapour saturation pressure at the droplet temperature, averaged over all droplets in the LES cell. Hence, the modelling of $\langle\Pi \mid \eta, \zeta\rangle_{\text {les }}$ is the equivalent of the $\delta$-function used in conventional CMC [28]. This presumed shape of the conditional spray term is scaled, such that $\langle\Pi \mid \eta, \zeta\rangle_{\text {les }}$ integrates with the FDF to give the filtered value $\widetilde{\Pi}$. As pointed out in previous work for singly-conditioned CMC [14, 27], this source term needs to be limited to avoid numerical instability in the case where the probability associated with $\left[Q_{\mathrm{F}}(\eta, \zeta)=\left\langle Y_{\mathrm{Fs}}\right\rangle_{\text {les }}\right]$ is very low. At the CMC resolution $\langle\Pi \mid \eta, \zeta\rangle_{\mathrm{cmc}}$ is obtained by volume averaging over the LES cells in the same way as for the conditional SDRs. $\left\langle S_{h} \mid \eta, \zeta\right\rangle_{\text {les }}$ is modelled using the same presumed shape as for $\langle\Pi \mid \eta, \zeta\rangle_{\text {les }}$, but it is scaled to integrate with the FDF to give $\tilde{S}_{h}$.

\subsection{Test case}

The test case studied in the present work is a lifted heptane spray jet flame open to the atmosphere. The burner is described in Refs [33, 34, 35]. It consists of a plenum and a nozzle without swirl. The exit of the nozzle has an annular shape with an inner and outer diameter of 10 and $20 \mathrm{~mm}$ respectively. The spray injector is located in the centre of the nozzle. The burner is operated with an air mass flow rate of $6 \mathrm{~g} / \mathrm{s}$, which gives a bulk velocity of $21.58 \mathrm{~m} / \mathrm{s}$ (at $p=1 \mathrm{~atm}$ and $T=298 \mathrm{~K}$ ) and a jet Reynolds number (based on the hydraulic diameter of the annulus) of about 13,900. The atomiser is a hollow-cone injector with a half-angle of $40^{\circ}$. At a distance of $10 \mathrm{~mm}$ from the injector, the spray has a Sauter mean diameter of $32 \mu \mathrm{m}$.

The flame is a test case of the Workshop on Turbulent Combustion of Sprays [52]. The experimental database contains Phase Doppler Anemomentry (PDA) measurements of the mean and the root mean square (RMS) of the droplet velocity and the diameter, OH Planar Laser Induced Fluorescence $(\mathrm{OH}-$ PLIF) imaging, measurements of the droplet temperature with Global Rainbow Refractometry Technique (GRT) [33, 34] and simultaneous high-speed OH-PLIF and high-speed Particle Image Velocimetry (PIV) [35. PDA data is available 
for a reacting and a non-reacting case. In this article only comparisons with the reacting case are shown.

\subsection{Numerical set-up}

This work used OpenFOAM-2.3.1 [53] with a flow field solver based on the

PIMPLE algorithm, interfaced with an in-house DCMC code. The Abramzon and Sirignano evaporation model [4] was implemented for OpenFOAM. The numerical domain is shown in Fig. 1 (left). It includes the complete nozzle and plenum of the burner. Downstream of the nozzle, the domain was of cylindrical shape with a diameter of $800 \mathrm{~mm}$ and a length of $500 \mathrm{~mm}$. A constant air mass flow rate was set as inlet boundary condition to the plenum. A slip wall boundary condition was applied at the sides of the cylindrical domain and a small co-flow of $0.1 \mathrm{~m} / \mathrm{s}$ was set around the outer dimensions of the burner (for $r>70 \mathrm{~mm}$ ). An unstructured tetrahedral LES mesh with 18M cells was used. It was refined in the nozzle and the region of the flame (Fig. 1) to a minimum size of $0.4 \mathrm{~mm}$. Four prism layers were applied to the walls in the nozzle with a thickness of the first layer of $0.045 \mathrm{~mm}$ corresponding to $y^{+} \approx 1.4$; the laminar sub-layer was resolved by three cells. The mesh fulfilled Pope's criterion for LES [44] in the whole region of interest resolving at least $95 \%$ of turbulent kinetic energy.

The spray injector was modelled as a hollow cone injection. A general volume distribution given by experimental results was used to sample droplets in the range $1<d<80 \mu \mathrm{m}$. The droplets were injected with a random half angle between $35^{\circ}$ and $50^{\circ}$ and an initial velocity in the range $25<U_{d}<33 \mathrm{~m} / \mathrm{s}$ depending on the initial diameter: $U_{d}=33 \mathrm{~m} / \mathrm{s}$ for small droplets $(d \leq 10 \mu \mathrm{m})$ decreasing linearly with diameter to $U_{d}=25 \mathrm{~m} / \mathrm{s}$ for large droplets $(d>55 \mu \mathrm{m})$. These choices were made so that the simulation matched the spray measurements at the first available location.

Besides the LES mesh, a coarser DCMC mesh with 644 cells, arranged in an O-grid was used. In the region of interest the DCMC cells were spaced with an increment of $2.5 \mathrm{~mm}$ in axial direction; 3 layers of cells were used in radial 
direction, assuring that the outer and the inner flame were resolved by different DCMC cells, and 7 cells were used in each circumference of the O-grid. At the inlets a set of conditional moments obtained from DCMC-0D (see section 3.1) corresponding to a weakly strained flame were set as the boundary condition. The same set of conditional moments was also used as the initial condition. Zero-gradient boundary conditions were set at the outlet and the walls.

The doubly-conditional space $D=\left\{(\eta, \zeta) \in \mathbb{R}^{2}: 0 \leq \eta \leq 1,0 \leq \zeta \leq 1\right\}$ was discretised with $71 \times 41$ nodes. The $\eta$-nodes were clustered in the flammable mixture fraction region and the $\zeta$-nodes were spaced progressively more closely for $\zeta$ approaching 1.

Numerical schemes for LES were of second order in space and first order in time; the time step was $10^{-6} \mathrm{~s}$. In the DCMC solver an operator splitting strategy, as discussed in Ref. [54], was pursued. Advective transport and diffusion in physical space were first- and second-order accurate respectively. In conditional space, first derivatives were implemented with an upwind scheme, second derivatives used a second order central differencing scheme. Transport in conditional space was integrated using the solver VODPK [55] and the chemical reactions were solved using the SpeedCHEM library [56]. The simulation used a complex chemical mechanism with 67 species and 315 elementary reactions [57, which does not include $\mathrm{NO}_{\mathrm{x}}$-chemistry. The simulation ran on a Cray XC30 system using $4322.7 \mathrm{GHz}$ processors; $1 \mathrm{~ms}$ of physical time took about 12,000 CPU hours on wall-clock time. Time-averages were collected over $10 \mathrm{~ms}$, corresponding to three flow-through times.

\section{Results and discussion}

Before presenting the LES-DCMC results for the test flame, it is instructive to conduct an a priori study using DCMC-0D with prescribed SDRs. First consider the DCMC equation in this simplified case and in absence of spray 
terms. Then Eqn. 15 becomes,

$$
\begin{aligned}
\frac{\partial Q_{\alpha}}{\partial t}= & \left\langle N_{\xi} \mid \eta, \zeta\right\rangle \frac{\partial^{2} Q_{\alpha}}{\partial \eta^{2}}+2\left\langle N_{\xi c} \mid \eta, \zeta\right\rangle \frac{\partial^{2} Q_{\alpha}}{\partial \eta \partial \zeta}+\left\langle N_{c} \mid \eta, \zeta\right\rangle \frac{\partial^{2} Q_{\alpha}}{\partial \zeta^{2}} \\
& +\left\langle\dot{\omega}_{\alpha} \mid \eta, \zeta\right\rangle-\left\langle\dot{\omega}_{c}^{*} \mid \eta, \zeta\right\rangle \frac{\partial Q_{\alpha}}{\partial \zeta}
\end{aligned}
$$

which is conceptually similar to the equation solved by Nguyen et al. [50]. A steady state solution of Eqn. 22 is used as initial condition for the DCMC solver and boundary condition at the inlets.

In this section, the solution from Eqn. 22 is compared to a two-dimensional manifold in $(\xi, c)$-space created from a set of one-dimensional freely propagating laminar premixed flames in the range of flammable mixture fractions. These laminar premixed flames were calculated using the commercial software Cosilab 58. For better comparison with the DCMC model, unity Lewis number was also imposed in the calculation of the laminar premixed flames. Extension of the DCMC model to include differential diffusion effects is not in the scope of the present study, but the suggestions made in Refs [59, 60] can be incorporated in future work.

In this a priori assessment the unscaled profile of the SDR from the onedimensional freely propagating laminar premixed flames $N_{c}^{0}(\eta, \zeta)$ (Fig. 2 was directly used to close $\left\langle N_{c} \mid \eta, \zeta\right\rangle$. For $\left\langle N_{\xi} \mid \eta, \zeta\right\rangle$ the AMC models's bell curve with $N_{\xi, \max }=2 \mathrm{~s}^{-1}$ was used - this is very small compared to its value at extinction $\approx 335 \mathrm{~s}^{-1}$. Both $\left\langle\dot{\omega}_{c} \mid \eta, \zeta\right\rangle$ and $Q_{T}=\langle T \mid \eta, \zeta\rangle$ agree well with $\dot{\omega}_{c}^{0}(\eta, \zeta)$ and $T^{0}(\eta, \zeta)$, respectively. Note that the shape of $Q_{\mathrm{CO} 2}=\left\langle Y_{\mathrm{CO} 2} \mid \eta, \zeta\right\rangle$ is fixed in time since the terms in its DCMC equation cancel each other. Thus, $Q_{\mathrm{CO} 2}$ automatically satisfies Eqn. 11 relating $\xi$ and $c$ with $Y_{\mathrm{CO} 2}$. Moreover, $Q_{\mathrm{N} 2}$ is fixed since $\mathrm{NO}_{\mathrm{x}}$ chemistry is not included in the chemical mechanism.

Second, consider the effect of the doubly-conditional spray source terms. The governing equation for the DCMC-0D problem then becomes

$$
\begin{aligned}
\frac{\partial Q_{\alpha}}{\partial t}= & {[\text { r.h.s. of Eqn. 22] }} \\
& +\left(\delta_{\alpha \mathrm{F}}-Q_{\alpha}\right)\langle\Pi \mid \eta, \zeta\rangle-\left\langle S_{\xi}^{-} \mid \eta, \zeta\right\rangle \frac{\partial Q_{\alpha}}{\partial \eta}-\left\langle S_{c}^{-} \mid \eta, \zeta\right\rangle \frac{\partial Q_{\alpha}}{\partial \zeta}
\end{aligned}
$$


Figure 3 shows a conditional evaporation term $\langle\Pi \mid \eta, \zeta\rangle$ for a droplet with a surface vapour mass fraction $Y_{\mathrm{Fs}}=0.65$, which is presumed as a ridge in $(\eta, \zeta)$ space following the suggestion of Sitte and Mastorakos [25]. In this example we further assume that $\left\langle S_{h} \mid \eta, \zeta\right\rangle=\langle\Pi \mid \eta, \zeta\rangle \times\left[-2.2 \cdot 10^{6} \mathrm{~W} / \mathrm{kg}\right]$; this is a typical ratio $\widetilde{S}_{h} / \widetilde{\Pi}$ found in the LES. It is notable that the net effect of the spray terms in $Q_{\alpha}$ is small if $Y_{\mathrm{Fs}}>\xi_{\text {rich }}$, where $Q_{\alpha}$ drops linearly towards its value at the boundary $\eta=1$ (note: the lean and rich flammability limits of n-heptane are $\xi_{\text {lean }} \approx 0.0339$ and $\xi_{\text {rich }} \approx 0.199661$ ). In the cases of $Q_{\mathrm{N} 2}$ and $Q_{\mathrm{CO} 2}$, which are unambiguously described by $\eta$ and $\zeta$, the three spray terms cancel each other completely, such that their shapes are never affected by evaporation. This is the same as in singly-conditional CMC.

In contrast, there is a strong net effect on $Q_{h}$ - this is the effect of evaporative cooling. Figure 3 shows that the first spray term $\left(\left\langle S_{h} \mid \eta, \zeta\right\rangle-Q_{h}\langle\Pi \mid \eta, \zeta\rangle\right)$ is partly compensated by the second one, $-\left\langle S_{\xi}^{-} \mid \eta, \zeta\right\rangle \partial Q_{h} / \partial \eta$. The third term, $-\left\langle S_{c}^{-} \mid \eta, \zeta\right\rangle \partial Q_{h} / \partial \zeta$, representing the effect of evaporation on $c$ is negligible by comparison. The net spray term is eventually counter-balanced by the SDR of the mixture fraction, which dissipates the evaporation source term and leads to the distortion of $Q_{h}$ shown relative to its initial shape $Q_{h}^{0}$. In the limit of steady state $Q_{h}$ decreases linearly in the interval $\eta \in\left[0, Y_{\mathrm{Fs}}\right]$. This suggests that the effect of evaporative cooling on the reaction zone, in terms of $\Delta h$ and $\Delta T$, is progressively smaller for hotter droplets when $Y_{\mathrm{Fs}}>\xi_{\text {rich }}$. Vice versa, cold droplets at first contact with the flame can be expected to have the largest effect on the flame.

\subsection{Flow field and spray statistics}

Figure 4 shows a good agreement of the mean axial velocity and its RMS from LES with PDA measurements for four axial locations. In Fig. 5 droplet diameter, temperature and velocity from LES are compared to PDA and GRT measurements. The good agreement between LES and the experimental data for the droplet velocity and diameter at the location $z=10 \mathrm{~mm}$ suggests that the modelling of the injector was suitable. Large droplets behave in a ballistic 
sense and significant slip velocities persist beyond $z=20$ for $r>15 \mathrm{~mm}$ and, in particular, at the flame anchoring point. Consequently, the flame benefits from enhanced evaporation to provide gaseous fuel (e.g. for a $30 \mu \mathrm{m}$ droplet with a 
attributed to low mean velocities in this region where the flame is laminar. Consequently, characteristic time scales for the outer flame are very long, $0.1 \mathrm{~s}$, as evidenced by $10 \mathrm{~Hz} \mathrm{OH}-\mathrm{PLIF}$ recordings, which is challenging to explore

outer and inner flame are visible. In the outer branch levels of $\mathrm{C}_{2} \mathrm{H}_{2}$ and $\mathrm{OH}$ are relatively higher, whereas the inner branch contains more $\mathrm{CH}_{2} \mathrm{O}$. In particular, 
the interaction of turbulence with the flame leads to regions of low $\widetilde{Y}_{\mathrm{OH}}$ in the inner flame brush, which was also observed in the experiment using high-speed

Furthermore, the results shown in Fig. 8 allow to comment on modelling choices. Only low $\tilde{Y}_{\mathrm{F}}=\tilde{Y}_{\mathrm{C} 7 \mathrm{H} 16}$ is found in the flame brush apart from the fuel pockets around droplets with strong evaporation rates. Conversely, the presence of pure fuel is expected near the surface of every droplet, even if this is not resolved by the LES. This is a sub-grid scale effect that requires special treatment of the spray terms in the $\widetilde{\xi^{\prime \prime 2}}$-equation discussed by Giusti and Mastorakos [14], which were not included in the present work. Future work on the sub-grid scale modelling of spray effects will be necessary.

In order to better understand the stabilisation of the flame, we consider the time-averaged fields shown in Fig. 9. The time-averaged mixture fraction $\langle\xi\rangle$ is very similar to the instantaneous one, with significant variance only occurring in the turbulent inner flame branch. In the outer flame branch the turbulent velocity fluctuation $u^{\prime}$ is very low, which shows that the flow is laminar in this region. The time-averaged fields indicate the presence of the dispersed phase, hence of liquid fuel and evaporation. Dashed black lines mark representative droplet trajectories and the region of highest liquid volume fraction $\left\langle\theta_{L}\right\rangle$ shows the dominant pathway of the droplets, crossing through the tip of the flame. Since the droplets behave in a ballistic way, moving relative to the gas phase (Fig. 5), they penetrate through the flame and the highest mean evaporation rate $\langle\rho \Pi\rangle$ occurs in the region of hot gases downstream of the tip of the flame. In contrast, the mean mixture fraction is very low in the cold gases upstream of the flame, as indicated by iso-contours. This shows that in the present case the flame itself "creates" the gaseous fuel required. Finally, Fig. 9 also reveals that the spray is indeed very dilute in the region occupied by the flame and that the highest average evaporation rate $\langle\rho \Pi\rangle$ is observed near the tip of the flame.

A detailed view of the flame's profile at its anchoring point can be obtained from Fig. 10. The profiles are drawn over the line indicated in Figs 8 and 9. perpendicular to the mean position of the flame. Primarily, these profiles 
highlight the low level of gaseous fuel available in the cold gases upstream of the

is observed. In this region, the existence of $\mathrm{O}_{2}$ suggests incomplete combustion.

Figure 11 (A) shows a cut through an instantaneous iso-surface of the stoichiometric mixture fraction. This reveals again large differences between the inner and outer branch of the flame. In general, temperature and $\tilde{Y}_{\mathrm{OH}}$ are lower at the inner flame brush but higher HRR indicates chemical reaction. Furthermore, the iso-contour is strongly wrinkled around the base of the flame due to the evaporation of droplets penetrating the flame. Typically, these are spots of lower temperature but are also surrounded by regions of high HRR.

It is notable that a wide range of temperature and species mass fractions is observed on the stoichiometric mixture fraction contour. In order to reproduce this result in conventional singly-conditional CMC a very large number of CMC cells would be required. In contrast, in the present DCMC approach the progress variable provides an additional degree of freedom that allows the resolution of small scale variations of temperature, HRR and species, using a moderate number of DCMC cells.

In Fig. 11 (B) a cut through two $\tilde{c}$ iso-contours $(\tilde{c}=0.1$ and 0.6$)$ shows that very little fuel evaporates upstream of the flame. Gaseous fuel is only generated by evaporation in response to the heat released by chemical reaction. Moreover, Fig. 11 highlights that most droplets penetrate through flame and continue to evaporate in the hot gases.

\subsection{DCMC versus constant flame structure}

In this section, the effect of solving the DCMC equation to capture the evolution of the flame structure is assessed. For this purpose, the LES-DCMC results 
are compared to an LES that uses a constant flame structure, invariant in space and time. This simulation uses the flame structure computed in the a priori assessment of DCMC-0D (Eqn. 22, instead of solving the DCMC equation. All other models and settings of the simulation are unchanged.

Figure 12 compares the results of the two simulations. The position of the flame and its shape are hardly affected. Both simulations show a lift-off hight of about $26 \mathrm{~mm}$. This suggests that the lift-off height of this particular flame appears to be determined by the spray injection and evaporation rather than the combustion model. The gas velocities and spray statistics are unchanged compared to Figs 4 and 5 (not shown). The fields of mean temperature from both simulations are overall similar, which explains the unchanged spray statistics and gas velocity.

The present flame is not exposed to very high levels of turbulence intensity and the SDR is relatively low. Hence, large differences due to the local variations in strain rate are not expected. Some differences can be observed at the turbulent inner flame branch, where LES-DCMC predicts higher mean temperature and mean HRR. Differences are also observed for most chemical species, in particular, for radical species. Here only $\mathrm{OH}$ mass fraction is shown, since a comparison to experimental OH-PLIF measurements is possible. LESDCMC predicts $\left\langle Y_{\mathrm{OH}}\right\rangle \approx 0.001$ in the inner flame brush, which is about three times higher than the mean $\mathrm{OH}$ in the simulation with constant flame structure. At the same time LES-DCMC predicts a lower level of $\mathrm{OH}$ in the outer flame branch. Hence, the LES-DCMC results appear to be more similar to the integrated OH-PLIF measurements that found comparable intensity in both flame branches (see Fig 7).

\subsection{Analysis in doubly-conditional space}

The previous analysis showed that the evolution of the doubly-conditional flame structure, solved in space and time by the DCMC equation, had a noticeable effect on the prediction of $\mathrm{OH}, \mathrm{HRR}$ and even temperature in the flame. 
The spatial and temporal evolution of the flame structure in DCMC is investigated next.

Figure 13 shows instantaneous doubly-conditional moments for three different locations, at the tip, the inner and the outer branch of the flame (see Fig. 11). Note that $Q_{\mathrm{CO} 2}$ (Fig. 2 ) is fixed in the entire domain according to the definition in Eqn. 1. At the locations A and B the conditional moments of temperature, $\mathrm{O} 2$ and $\mathrm{OH}$ are relatively similar. Using the doubly-conditional parametrisation, the conditional means of major species seem to be less sensitive to location than they are in singly-conditional CMC. At the same time, significant differences persist for the reaction rates, suggesting that the balance of minor species are very sensitive to location. Larger differences are observed when comparing the inner and outer flame branch (locations B and D).

The balance of terms in the DCMC equation is analysed here focusing on the radical species $\mathrm{OH}$. It was shown previously that solving for the evolution of the flame structure with DCMC has an effect on the prediction of $\mathrm{OH}$ in the inner flame branch. Figure 14 shows the instantaneous terms of the DCMC equation for $\mathrm{OH}$ at the location of the anchoring point of the flame (location A). The conditional moment $Q_{\mathrm{OH}}$ itself and $\left\langle\dot{\omega}_{c} \mid \eta, \zeta\right\rangle$ are displayed in Fig. 13 (location A). In contrast to singly-conditional CMC (for instance, Fig. 11 in Ref. [62]), the present case, the terms representative of transport in physical space, advection, dilatation and diffusion, do not play an important role in the balance of terms. This is in line with the reduced sensitivity of $Q_{\mathrm{OH}}$ to spatial location and relatively lower gradients in physical space, as compared to singly-conditional CMC. This can be related partly to the fact that this flame does not exhibit a very strong transient behaviour, such that the flame structure parametrised in doubly-conditional space evolves only gradually in space. However, more generally, the progress variable provides an additional degree of freedom in conditional space that can be seen as representative for the spatial evolution across the flame. The reduced sensitivity of certain doublyconditional moments on location has recently been discussed by Bushe 63.

Amongst the terms of transport in physical space the diffusion term is more 
than one order of magnitude smaller than the advective term (not shown) since singly-conditional CMC. This is, in particular, true for major species and temperature. The instantaneous reaction rate is more strongly affected by small changes in SDR and, consequently, large variations are observed both in terms of spatial differences and temporal fluctuations. This is summarised in Fig. 15 , action rate for different locations. First, $\left\langle\dot{\omega}_{c} \mid \eta, \zeta\right\rangle$ is investigated at the locations $\mathrm{A}, \mathrm{B}$ and $\mathrm{C}$, which distinguish each other in terms of their axial distance from the nozzle (Fig. 1). Moreover, time-averages of the conditional reaction rate $\left\langle\left\langle\dot{\omega}_{c} \mid \eta, \zeta\right\rangle\right\rangle$ and the corresponding RMS are shown. At location D, in the outer 
action rate at $\mathrm{D}$ is identical with the instantaneous one shown in Fig. 13.

Since the highest SDRs occur close to the tip of the flame (Fig. 8), the region around location $\mathrm{A}$ experiences the highest strain and, thus, exhibits the lowest conditional reaction rate. For all three locations the RMS values are for fluctuations differ significantly: while they are very fast at location $\mathrm{A}$, the flame around point $\mathrm{C}$ experiences slow transitions over several milliseconds. Secondly, the conditional reaction rate in various locations with identical radial and axial coordinates but at different azimuthal locations is studied; A1, A2, occupy consecutive azimuthal positions. In spite of the burner's geometry, the test flame only exhibits rotational symmetry in the mean sense. Consequently, the behaviour in neighbouring azimuthal locations is similar, but significant differences in conditional reaction rate persist at most instances in time.

This analysis demonstrates the following points: (i) gradients of the conditional means in physical space are greatly reduced by the doubly-conditional parametrisation of the flame structure; (ii) still, important spatial differences and temporal evolutions of the conditional means of minor species and reaction rates persist on top of the doubly-conditional parametrisation, even in the relatively stable test flame studied here; and (iii) this underlines the necessity for a sufficient resolution in terms of DCMC cells.

\section{Conclusions}

In this work we presented an application of the LES-DCMC modelling approach. Doubly-conditional spray terms have been included in the DCMC equation to introduce the effect of evaporation on the reaction zone. The present formulation of DCMC based on mixture fraction and reaction progress variable allows to capture both premixed and non-premixed burning modes that may co-exist in many flames and, in particular, in spray combustion. 
In an a priori assessment the DCMC model, given the right conditional SDRs, successfully reproduced the conditional reaction rate and temperature from freely propagating laminar premixed flames. This confirms the validity of the DCMC model in this limit case and supports the choice of sub-models used for the doubly-conditional SDR of reaction progress variable.

LES-DCMC was employed to simulate a lifted spray jet flame using a detailed chemical mechanism for n-heptane fuel. The study found good agreement between simulation results and experiments in terms of instantaneous as well as time-averaged flame shape and droplet statistics, hence validating the LESDCMC approach for spray flames. The simulation allows for a detailed analysis of the flame and its anchoring point.

The results from LES-DCMC were compared to a second LES simulation that used a space- and time-invariant flame structure. While both simulations predicted the same lift-off height, some differences were found for OH, HRR and temperature in the turbulent inner flame branch.

The analysis of the terms in the DCMC equation suggested that non-premixed burning modes are prominent at the anchoring point of the flame. Hence, the doubly-conditional description of the flame can be used as a metric to gain insight into the driving mechanism behind the flame. Furthermore, it was found that advective and diffusive transport are less important in DCMC than in the conventional singly-conditional CMC. This is attributed to the fact, that the additional dimension in conditional space reduces the dependency of the flame structure on physical space and the gradients of the doubly-conditional moments in physical space are lower. Analysing iso-contours of stoichiometric mixture fraction showed that the doubly-conditional parametrisation of the flame also allowed to resolve local effects due to droplet evaporation, which again supports the use of DCMC for spray flames.

While conditional moments of temperature and major species are similar in large parts of the domain, the conditional reaction rate has a significant dependence on the location in the flame. Moreover, it exhibits temporal fluctuations of about $10 \%$ of its local mean, whose time scales depend on the location. 

flame structure, a very coarse DCMC mesh may be sufficient to capture strongly transient effects. Therefore, the DCMC approach may have the potential for increased computational efficiency compared to conventional CMC, which should be assessed in future work. In terms of modelling future work should focus on (i) differential diffusion in DCMC, (ii) the further validation of conditional spray source terms, only considered in a small number of publications so far, and (iii) the modelling of sub-grid scale evaporation effects, in particular, on mixture fraction variance, FDF and SDR.

\section{Acknowledgements}

We would like to acknowledge useful discussions with Dr A. Giusti and we are thankful to Mr P. Benie for his help with the use of computational resources. M.P. Sitte gratefully acknowledges funding from the Gates Cambridge Trust. Experimental data used for comparison with the simulation results was kindly provided by Dr B. Renou. This work used the ARCHER UK National Supercomputing Service http://www.archer.ac.uk with computational time provided by the UK Consortium on Turbulent Reacting Flows.

\section{References}

[1] P. Jenny, D. Roekaerts, N. Beishuizen, Modeling of turbulent dilute spray combustion, Prog. Energ. Combust. Sci. 38 (2012) 846-887.

[2] W. A. Sirignano, Fuel droplet vaporization and spray combustion theory, Prog. Energ. Combust. 9 (1983) 291-322.

[3] J. Réveillon, L. Vervisch, Analysis of weakly turbulent dilute-spray flames and spray combustion regimes, J. Fluid Mech. 537 (2005) 317-347.

[4] G. Myers, A. Lefebvre, Flame propagation in heterogeneous mixtures of fuel drops and air, Combust. Flame 66 (1986) 193-210. 
[5] E. Mastorakos, Forced ignition of turbulent spray flames, Proc. Combust. Inst. 36 (2) (2017) 2367-2383.

[6] R. Yuan, J. Kariuki, E. Mastorakos, Measurements in swirling spray flames at blow-off, Int. J. Spray Combust. (2018) 1-26.

[7] A. Y. Klimenko, R. W. Bilger, Conditional moment closure for turbulent combustion, Prog. Energ. Combust. 25 (1999) 595-687.

[8] A. Kronenburg, E. Mastorakos, The Conditional Moment Closure Model, in: T. Echekki, E. Mastorakos (Eds.), Turbulent Combustion Modelling, Springer, 2011.

[9] S. Navarro-Martinez, A. Kronenburg, F. D. Mare, Conditional moment closure for large eddy simulations, Flow Turbul. Combust. 75 (1-4) (2005) $245-274$.

[10] A. Triantafyllidis, E. Mastorakos, R. L. G. M. Eggels, Large eddy simulations of forced ignition of a non-premixed bluff-body methane flame with conditional moment closure, Combust. Flame 156 (12) (2009) 2328-2345.

[11] S. Navarro-Martinez, A. Kronenburg, Flame stabilization mechanisms in lifted flames, Flow, Turbulence and Combustion 87 (2) (2011) 377-406.

[12] H. Zhang, A. Garmory, D. E. Cavaliere, E. Mastorakos, Large eddy simulation/conditional moment closure modeling of swirl-stabilized non-premixed flames with local extinction, Proc. Combust. Inst. 35 (2015) 1167-1174.

[13] H. Zhang, E. Mastorakos, Prediction of global extinction conditions and dynamics in swirling non-premixed flames using LES/CMC modelling, Flow Turbul. Combust. 96 (2016) 863-889.

[14] A. Giusti, E. Mastorakos, Detailed chemistry LES/CMC simulation of a swirling ethanol spray flame approaching blow-off., Proc. Combust. Inst. 36 (2017) 2625-2632. 
[15] D. Farrace, K. Chung, S. S. Pandurangi, Y. M. Wright, K. Boulouchos, N. Swaminathan, Unstructured LES-CMC modelling of turbulent premixed bluff body flames close to blow-off, Proc. Combust. Inst. 36 (2017) 19771985.

[16] P. Domingo, L. Vervisch, J. Réveillon, DNS analysis of partially premixed combustion in spray and gaseous turbulent flame-bases stabilized in hot air, Combust. Flame 140 (2005) 172-195.

[17] A. R. Masri, J. D. Gounder, Turbulent spray flames of acetone and ethanol approaching extinction, Combust. Sci. Technol. 182 (4-6) (2010) 702-715.

[18] J. Kariuki, E. Mastorakos, Experimental investigation of turbulent flames in uniform dispersions of ethanol droplets, Combust.Flame 179 (2017) 95116.

[19] D. H. Wacks, N. Chakraborty, Flame structure and propagation in turbulent flame-droplet interaction: A direct numerical simulation analysis, Flow Turbul. Combust. 96 (4) (2016) 1053-1081.

[20] D. H. Wacks, N. Chakraborty, E. Mastorakos, Statistical analysis of turbulent flame-droplet interaction: A direct numerical simulation study, Flow Turbul. Combust. 96 (2) (2016) 573-607.

[21] C. M. Cha, G. Kosály, H. Pitsch, Modeling extinction and reignition in turbulent nonpremixed combustion using a doubly-conditional moment closure approach, Phys. Fluids 13 (12) (2001) 3824-3834.

[22] A. Kronenburg, Double conditioning of reactive scalar transport equations in turbulent nonpremixed flames, Phys. Fluids 16 (2004) 2640-2648.

[23] A. Kronenburg, A. Papoutsakis, Conditional moment closure modeling of extinction and re-ignition in turbulent non-premixed flames, Proceedings of the Combustion Institute 30 (1) (2005) $759-766$. 
[24] F. Salehi, M. Talei, E. R. Hawkes, A. Bhagatwala, J. H. Chen, C. S. Yoo, S. Kook, Doubly conditional moment closure modelling for HCCI with temperature inhomogeneities, Proc. Combust. Inst. 36 (3) (2017) 3677 3685 .

[25] M. P. Sitte, E. Mastorakos, Modelling of spray flames with doubly conditional moment closure, Flow Turbul. Combust. 99 (3) (2017) 933-954.

[26] D. Dovizio, J. W. Labahn, C. B. Devaud, Doubly conditional source-term estimation (DCSE) applied to a series of lifted turbulent jet flames in cold air, Combust. Flame 162 (5) (2015) 1976 - 1986.

[27] G. Borghesi, E. Mastorakos, C. B. Devaud, R. W. Bilger, Modeling evaporation effects in conditional moment closure for spray autoignition, Combust. Theor. Model. 15 (2011) 725-752.

[28] A. Tyliszczak, D. E. Cavaliere, E. Mastorakos, LES/CMC of blow-off in a liquid fueled swirl burner, Flow Turbul. Combust. 92 (2014) 237-267.

[29] A. Giusti, M. Kotzagianni, E. Mastorakos, LES/CMC simulations of swirlstabilised ethanol spray flames approaching blow-off, Flow. Turbul. Combust. 97 (2016) 1165-1184.

${ }_{685}$ [30] S. Ukai, A. Kronenburg, O. Stein, LES-CMC of a dilute acetone spray flame, Proc. Combust. Inst. 34 (2013) 1643-1650.

[31] S. Ukai, A. Kronenburg, O. T. Stein, Simulation of dilute acetone spray flames with LES-CMC using two conditional moments, Flow Turbul. Combust. 93 (2014) 405-423.

[32] S. Ukai, A. Kronenburg, O. T. Stein, Large eddy simulation of dilute acetone spray flames using CMC coupled with tabulated chemistry, Proc. Combust. Inst. 35 (2015) 1667-1674.

[33] F. Shum-Kivan, J. M. Santiago, A. Verdier, E. Riber, B. Renou, G. Cabot, B. Cuenot, Experimental and numerical analysis of a turbulent spray flame structure, Proc. Combust. Inst. 36 (2) (2017) 2567-2575. 
[34] A. Verdier, J. M. Santiago, A. Vandel, S. Saengkaew, G. Cabot, G. Grehan, B. Renou, Experimental study of local flame structures and fuel droplet properties of a spray jet flame, Proc. Combust. Inst. 36 (2) (2017) 25952602.

[35] A. Verdier, J. M. Santiago, A. Vandel, G. Godard, G. Cabot, B. Renou, Local extinction mechanisms analysis of spray jet flame using high speed diagnostics, Combustion and Flame 193 (2018) 440-452.

[36] D. Noh, S. Gallot-Lavallée, W. P. Jones, S. Navarro-Martinez, Comparison of droplet evaporation models for a turbulent, non-swirling jet flame with a polydisperse droplet distribution, Combustion and Flame 194 (2018) 135151.

[37] A. Chatelier, V. Moureau, N. Bertier, B. Fiorina, Large eddy simulation of a spray jet flame using filtered tabulated chemistry, Proc. Tenth Mediterranean Combustion Symposium, Naples, Italy (2017) 1-11.

[38] A. Both, RANS-FGM simulation of n-heptane spray flame in OpenFOAM, MSc Thesis (2017).

1 URL https://repository.tudelft.nl/islandora/object/uuid: 0851a55a-4490-4176-a587-20b9585c09be?collection=education

[39] K. Bray, P. Domingo, L. Vervisch, Role of the progress variable in models for partially premixed turbulent combustion, Combust. Flame 141 (2005) 431-437.

[40] C. Fureby, On subgrid scale modeling in large eddy simulations of compressible fluid flow, Phys. Fluids 8 (5) (1996) 1301-1311.

[41] A. Garmory, E. Mastorakos, Capturing localised extinction in Sandia Flame F with LES-CMC, Proc. Combust. Inst. 33 (1) (2011) 1673-1680.

[42] T. Dunstan, Y. Minamoto, N. Chakraborty, N. Swaminathan, Scalar dissipation rate modelling for large eddy simulation of turbulent premixed flames, Proc. Combust. Inst. 34 (1) (2013) 1193 - 1201. 
[43] Z. Chen, S. Ruan, N. Swaminathan, Large eddy simulation of flame edge evolution in a spark-ignited methane-air jet, Proc. Combust. Inst. 36 (2) (2017) 1645-1652.

[44] S. B. Pope, Turbulent Flows, Cambridge University Press, 2000.

[45] I. Langella, N. Swaminathan, Y. Gao, N. Chakraborty, Large eddy simulation of premixed combustion: Sensitivity to subgrid scale velocity modeling, Combust. Sci. Technol. 189 (1) (2017) 43-78.

[46] L. Schiller, A. Z. Naumann, Über die grundlegende Berechnung bei der Schwerkraftaufbereitung, Zeitschift des Vereins Deutscher Ingenieure 77 (1933) 318-320.

[47] B. Abramzon, W. A. Sirignano, Droplet vaporization model for spray combustion calculations, Int. J. Heat Mass Transfer 32 (1989) 1605-1618.

[48] M. Mortensen, R. W. Bilger, Derivation of the conditional moment closure equations for spray combustion, Combust. Flame 156 (2009) 62-72.

[49] A. Garmory, E. Mastorakos, Numerical simulation of oxy-fuel jet flames using unstructured LES-CMC, Proc. Combust. Inst. 35 (2015) 1207-1214.

[50] P.-D. Nguyen, L. Vervisch, V. Subramanian, P. Domingo, Multidimensional flamelet-generated manifolds for partially premixed combustion, Combust. Flame 157 (2010) 43-61.

[51] E. E. O'Brien, T.-L. Jiang, The conditional dissipation rate of an initially binary scalar in homogeneous turbulence, Phys. Fluids A 3 (1991) 31213123.

[52] Workshop on Measurement and Computation of Turbulent Spray Combustion, www.tcs-workshop.org.

[53] The OpenFOAM Foundation Ltd, OpenFOAM-2.3.1, https://openfoam. org/ (2014). 
[54] Y. M. Wright, G. D. Paola, K. Boulouchos, E. Mastorakos, Simulations of spray autoignition and flame establishment with two-dimensional CMC, Combust. Flame 143 (2005) 402-419.

[55] A. C. Hindmarsh, Serial Fortran solvers for ODE initial value problems, https://computation.llnl.gov/casc/odepack/odepack_home.html (2006).

[56] F. Perini, The SpeedCHEM project, http://www.federicoperini.info/ speedchem (2013).

[57] A. Smallbone, W. Liu, C. Law, X. You, H. Wang, Experimental and modeling study of laminar flame speed and non-premixed counterflow ignition of n-heptane, Proc. Combust. Inst. 32 (1) (2009) 1245-1252.

[58] Rotexo GmbH and Co. KG, Cosilab Collection, Version 3.3.2, http://www. rotexo.com (2012).

[59] A. Kronenburg, R. W. Bilger, Modelling of differential diffusion effects in nonpremixed nonreacting turbulent flow, Phys. Fluids 9 (5) (1997) 14351447.

[60] D. Farrace, K. Chung, M. Bolla, Y. M. Wright, K. Boulouchos, E. Mastorakos, A LES-CMC formulation for premixed flames including differential diffusion, Combust. Theor. Model. 22 (3) (2018) 411-431.

[61] H. F. Coward, G. W. Jones, Limits of flammability of gases and vapors, Bureau of Mines Bulletin 503.

[62] H. Zhang, E. Mastorakos, Modelling local extinction in Sydney swirling non-premixed flames with LES/CMC, Proc. Combust. Inst. 36 (2) (2017) $1669-1676$.

[63] W. Bushe, Spatial gradients of conditional averages in turbulent flames, 

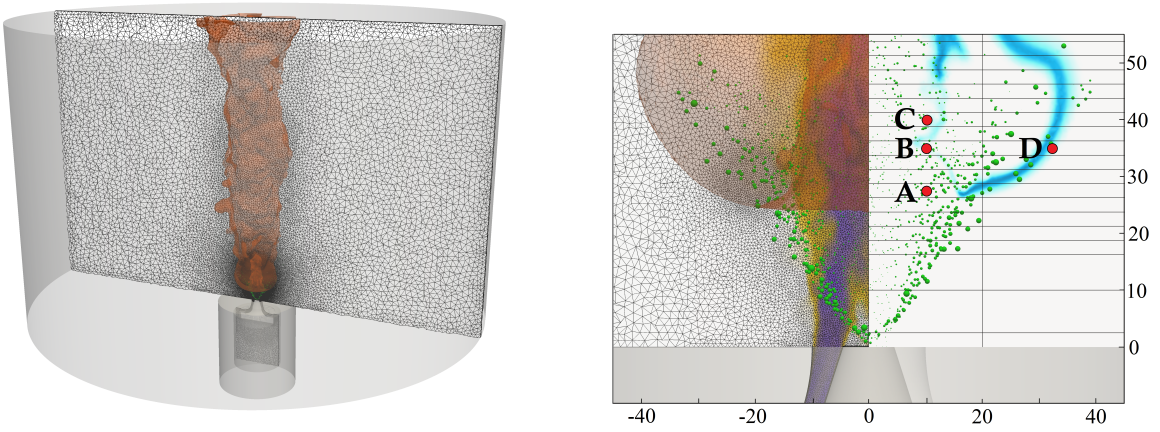

Figure 1: Numerical domain (left) and detailed view of the region of interest (right). The position of the flame is indicated by the red $\tilde{c}=0.1$ iso-contour. The detailed view shows the LES mesh (left half) and outlines of DCMC cells (right half). Additionally, droplets and contours of axial velocity and $\mathrm{OH}$ mass fraction are shown. The locations $\mathrm{A}$ at $(r, z)=$ $(10 \mathrm{~mm}, 27.5 \mathrm{~mm}), \mathrm{B}$ at $(10 \mathrm{~mm}, 35 \mathrm{~mm}), \mathrm{C}$ at $(10 \mathrm{~mm}, 40 \mathrm{~mm})$ and $\mathrm{D}$ at $(30 \mathrm{~mm}, 35 \mathrm{~mm})$ are selected for detailed analysis.
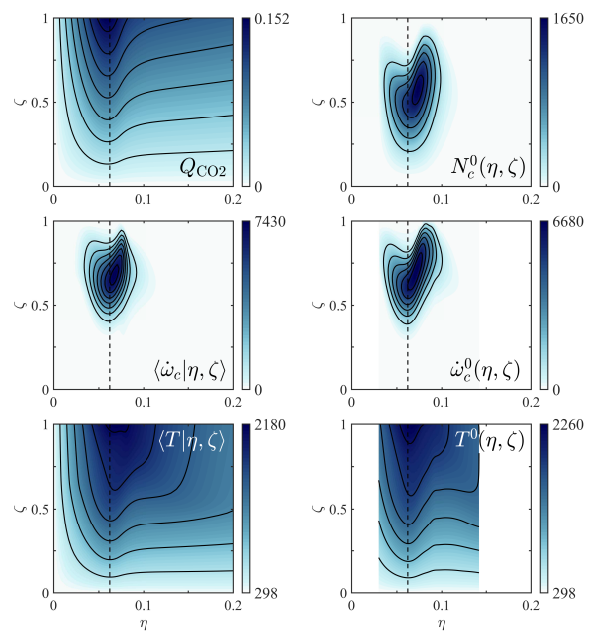

Figure 2: Comparison of temperature and reaction rate from a steady-state solution from DCMC-0D, $\left\langle\dot{\omega}_{c} \mid \eta, \zeta\right\rangle$ and $\langle T \mid \eta, \zeta\rangle$, with conditional values obtained from freely propagating laminar premixed flame calculations, $\dot{\omega}_{c}^{0}(\eta, \zeta)$ and $T^{0}(\eta, \zeta) . N_{c}^{0}(\eta, \zeta)$ is the conditional SDR from freely propagating premixed flames used as input to the DCMC-0D equation. The dashed vertical line marks the stoichiometric mixture fraction $\xi_{\text {st }} \approx 0.0622$. 

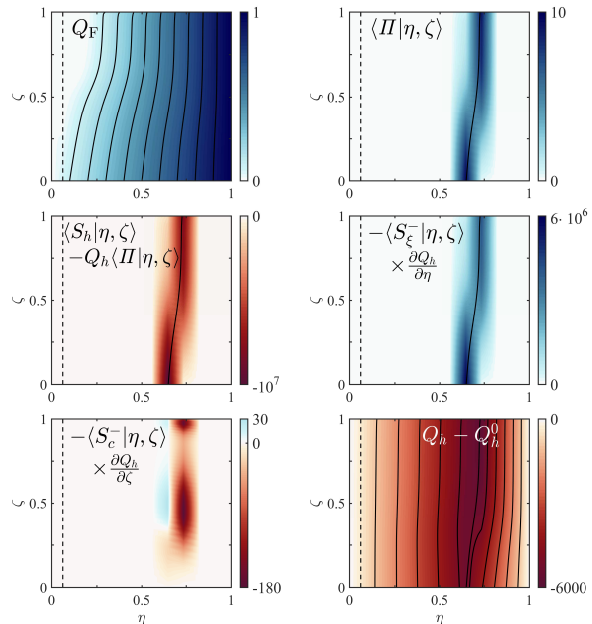

Figure 3: Steady-state solution of the DCMC-0D problem for $Q_{h}$, relative to the case without spray terms denoted as $Q_{h}^{0}$. The dashed vertical line marks $\xi_{\text {st }}$.

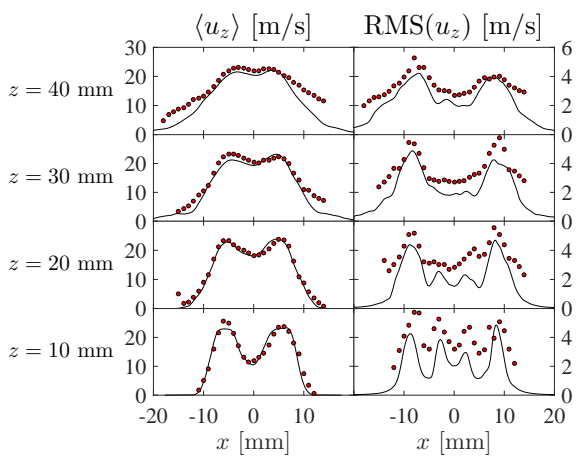

Figure 4: Profiles of axial velocity. Mean and RMS from LES (line) are compared to PDA measurements [33] (symbols) for the reacting case at four axial locations. 

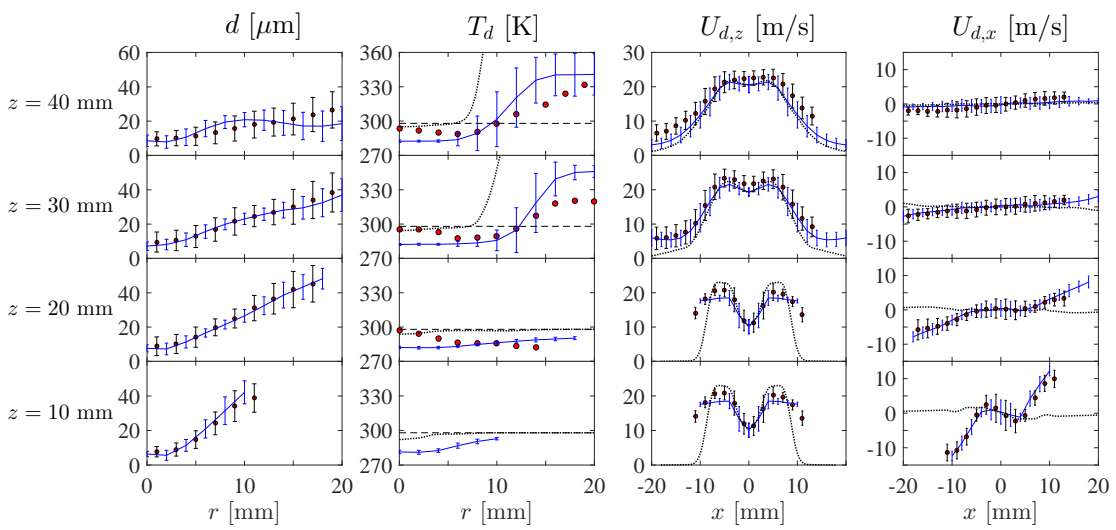

Figure 5: Profiles of mean diameter, droplet temperature and droplet velocity from LES (blue line) compared to PDA [33] and GRT 34] measurements (symbols) for four axial locations. Error bars represent the RMS value from LES and experiment at a given location (no RMS was available for GRT measurements). Black dotted lines indicate the mean gas velocity and mean gas temperature in the respective graphs. Black dashed lines mark the initial droplet temperature $298 \mathrm{~K}$.
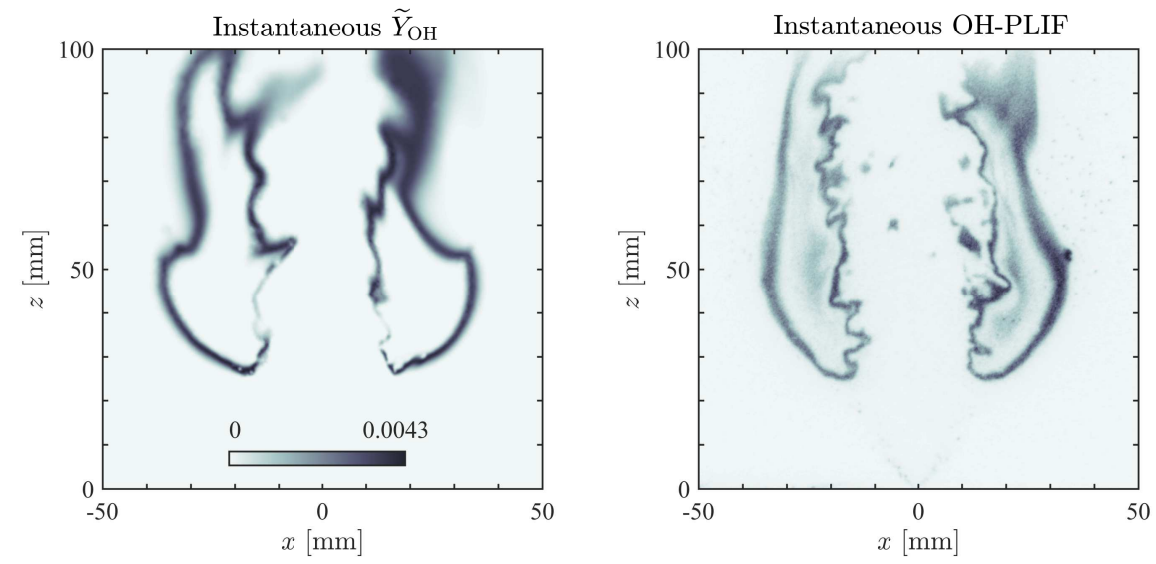

Figure 6: Instantaneous field of $\widetilde{Y}_{\mathrm{OH}}$ from LES (left) compared to instantaneous OH-PLIF intensity from the experiment 33 (right). 

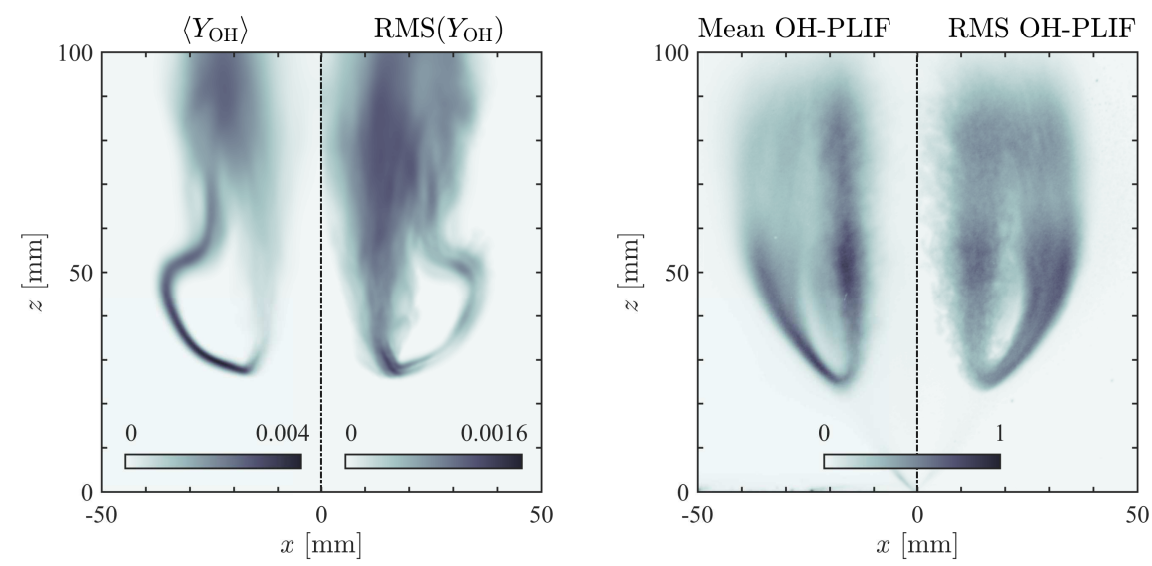

Figure 7: Time-averaged $\mathrm{OH}$ mass fraction $\left\langle Y_{\mathrm{OH}}\right\rangle$ and corresponding RMS from LES (left) compared to time-averaged OH-PLIF intensity and its RMS from the experiment 33] (right). For mean OH-PLIF and its RMS the same colour map is used. 

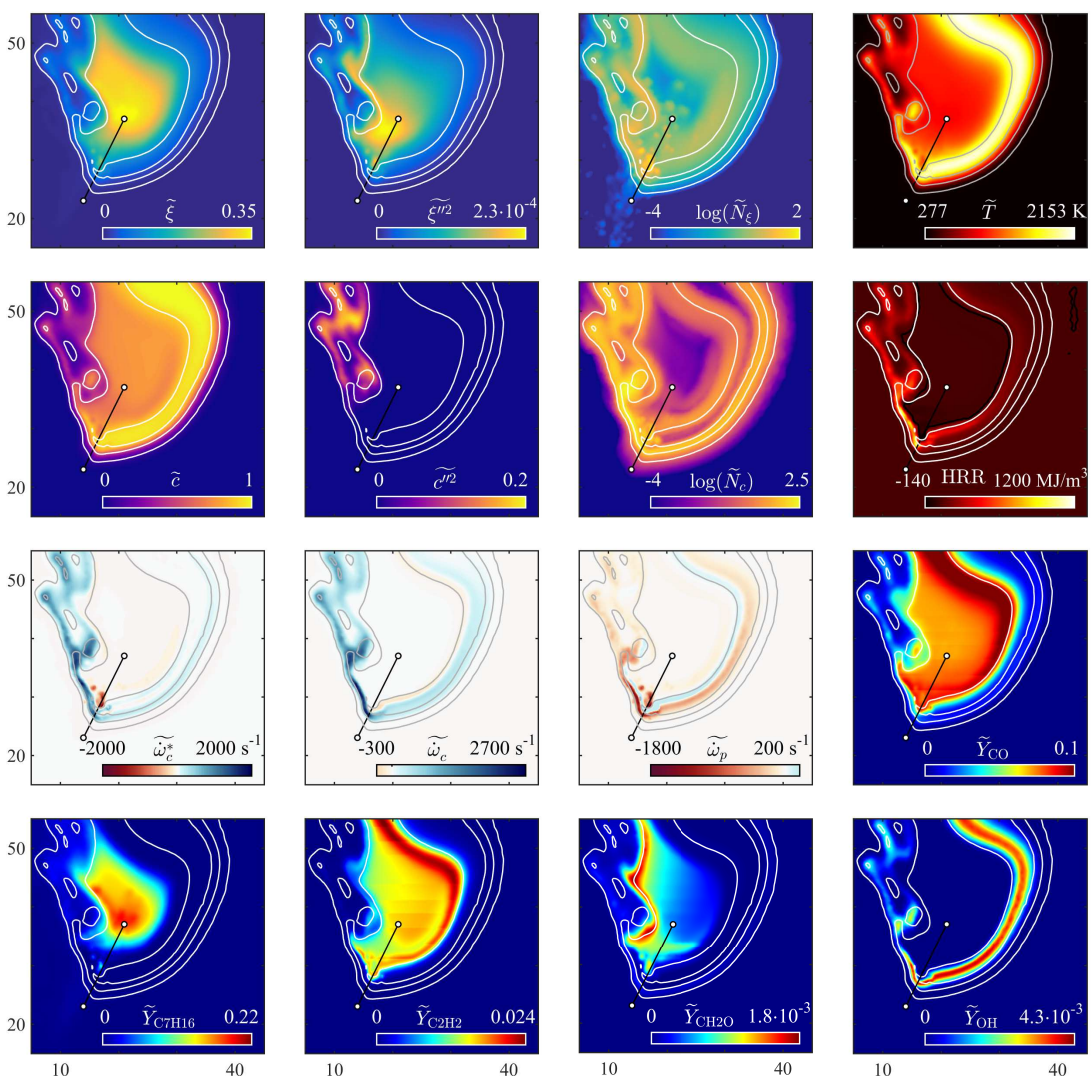

Figure 8: Instantaneous fields for various LES-filtered quantities. White (grey in the case of a light background) contour lines are iso-lines of $\tilde{c}=0.1,0.5,0.9$. The SDRs are given in $\mathrm{s}^{-1}$, logarithmic scales are clipped at -4 . Thick, black lines mark the areas of negative HRR in the respective image. A black line between white circles marks the orientation of the cut used in Fig. 10 

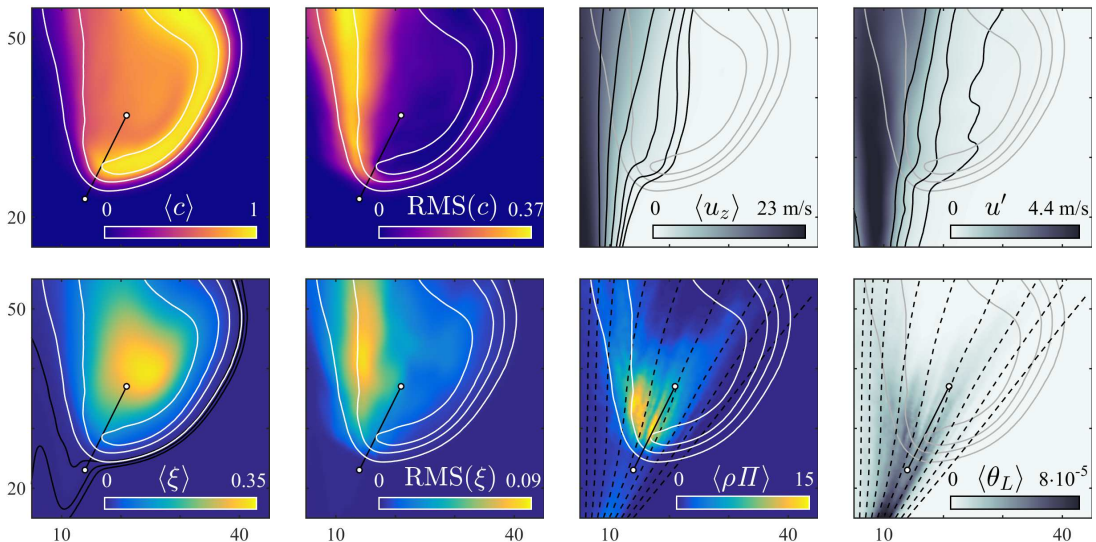

Figure 9: Time-averaged fields of various LES-filtered quantities. White (grey in images with light background) iso-lines are for $\langle c\rangle=0.1,0.5$ and 0.9 ; solid black iso-lines are for mean axial velocity $\left\langle u_{z}\right\rangle=0.5,1,2,5,10,15 \mathrm{~m} / \mathrm{s}$, turbulent velocity fluctuation $u^{\prime}=0.2,0.5,1,2$, and mean mixture fraction $\langle\xi\rangle=0.001,0.002,0.004$, in the respective images. Dashed black lines show representative droplet trajectories. A black line between white circles marks the orientation of the cut used in Fig. 10

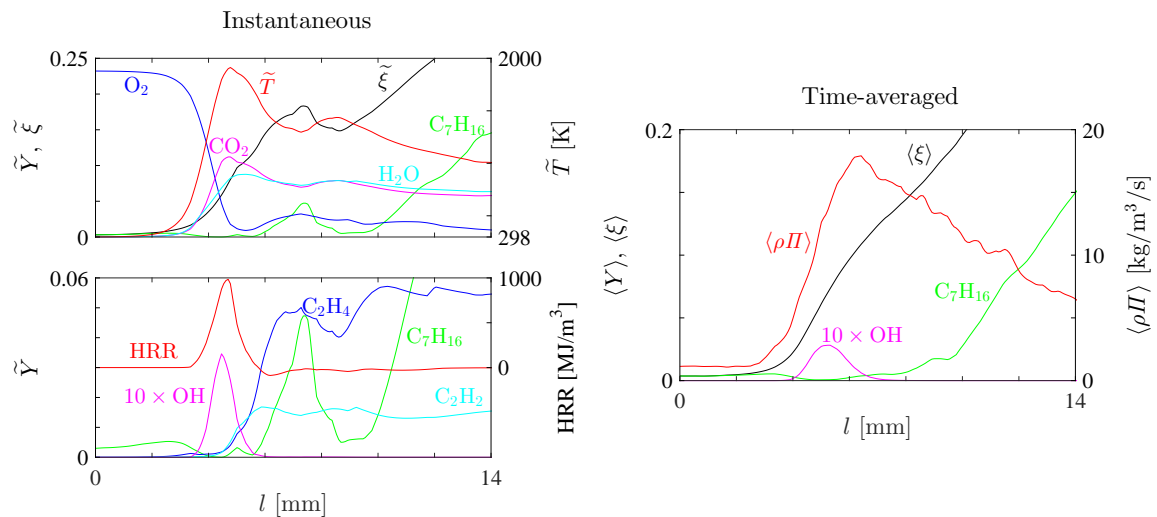

Figure 10: Instantaneous and time-averaged profile of the flame through the anchoring point, perpendicular to the $\langle c\rangle=0.5$ iso-contour. 
(A)

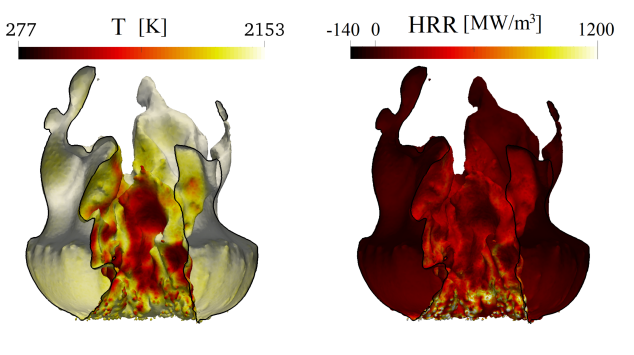

(B)
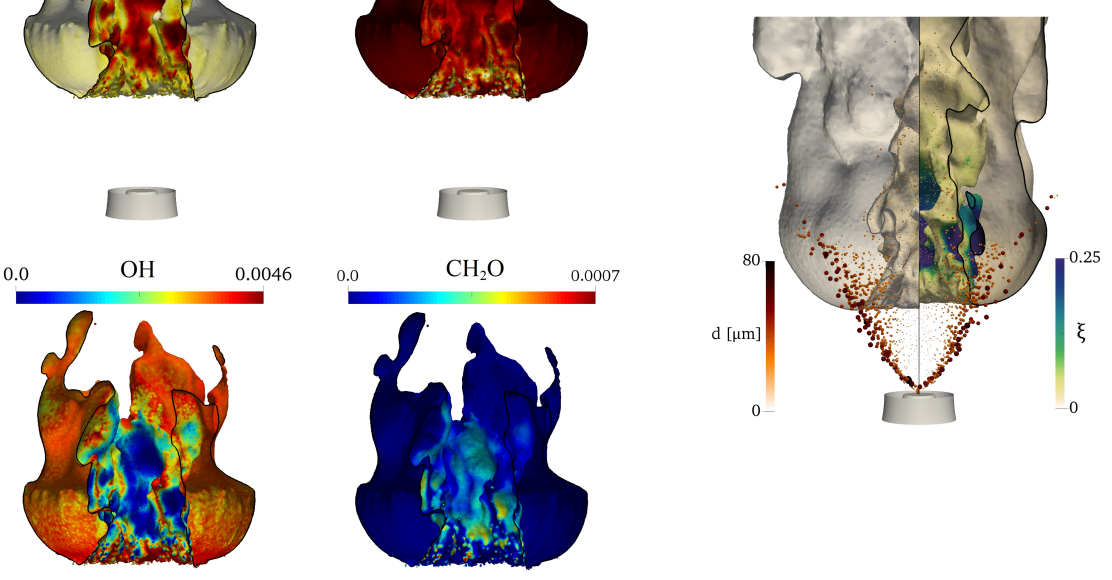

Figure 11: (A) Cut through the stoichiometric mixture fraction iso-surface; coloured by LESfiltered temperature, heat release rate and mass fractions of $\mathrm{OH}$ and $\mathrm{CH}_{2} \mathrm{O}$. The contour in the cut-plane is indicated by a black iso-line. (B) Cut through the reaction progress variable iso-surfaces $\tilde{c}=0.1$ (left) and $\tilde{c}=0.6$ (right), coloured by mixture fraction; the colour of droplets indicates their size. 

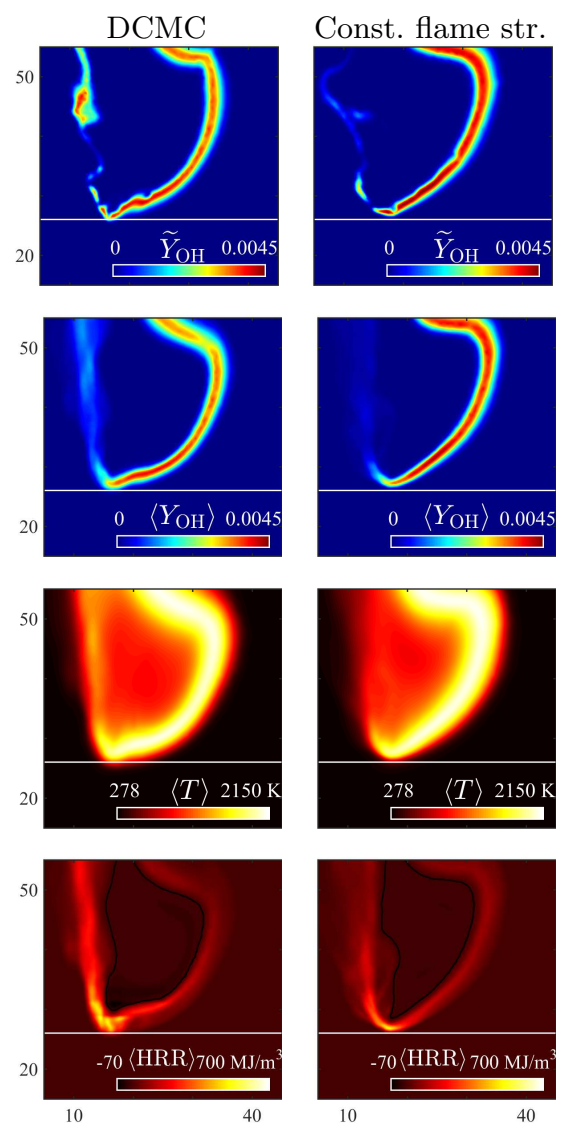

Figure 12: Comparison of results from LES-DCMC (left) and LES with space- and timeinvariant flame structure (right), for instantaneous $\tilde{Y}_{\mathrm{OH}}$ and the time-averaged fields $\left\langle Y_{\mathrm{OH}}\right\rangle$, $\langle T\rangle$ and $\langle$ HRR $\rangle$. The white horizontal line at $z=26 \mathrm{~mm}$ indicates the lift-off height obtained from LES-DCMC. A black line encompasses the region with negative mean HRR in the respective plots. 

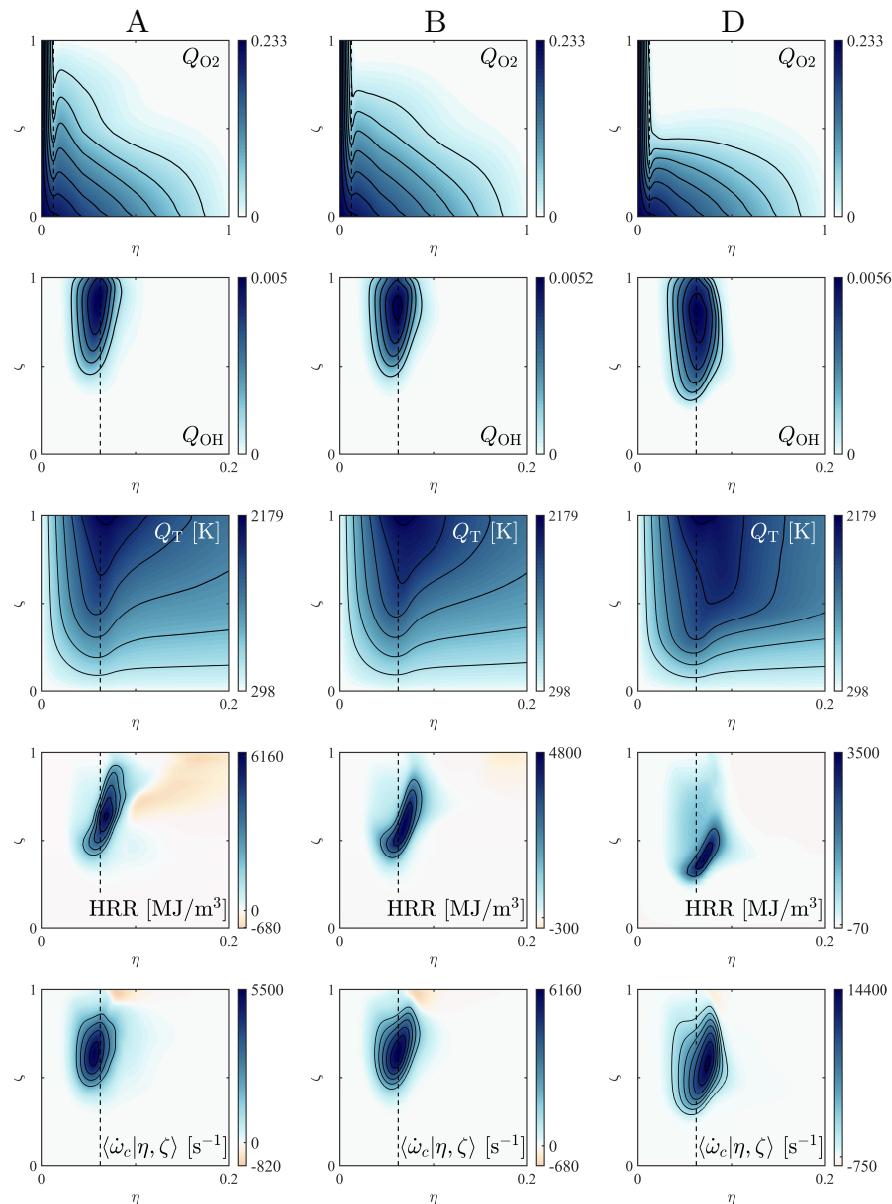

Figure 13: Instantaneous doubly-conditional moments from the tip of the flame at location A (left), the inner flame branch at location B (middle) and the outer flame branch at location $\mathrm{D}$ (right). The dashed vertical line marks $\xi_{\text {st. }}$. 

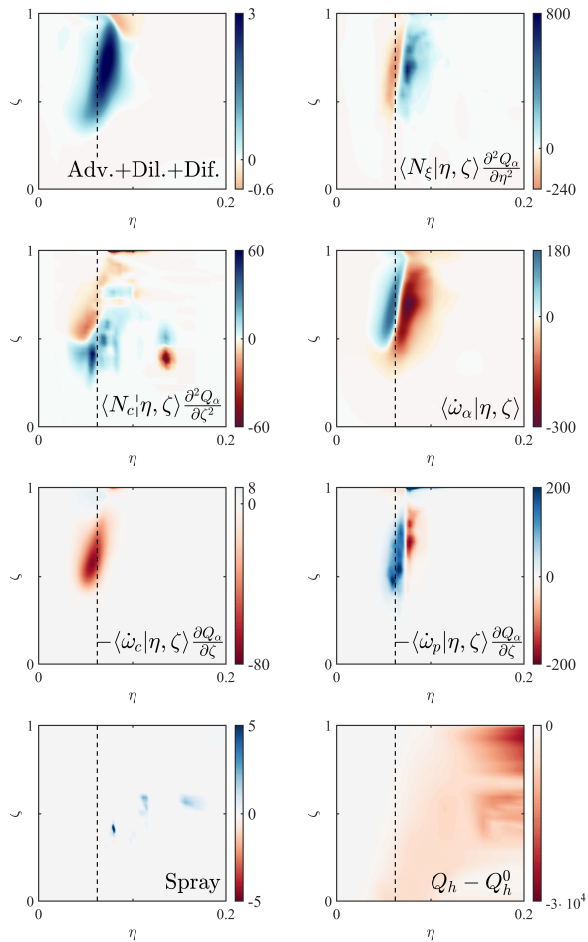

Figure 14: Instantaneous term balance for the DCMC equation of $Q_{\mathrm{OH}}$ for the CMC cell at location A $(r, z)=(10 \mathrm{~mm}, 27.5 \mathrm{~mm})$. Also shown is $Q_{h}-Q_{h}^{0}$, representing the net effect of the spray term on $Q_{h}$. The dashed vertical line marks $\xi_{\text {st }}$. 

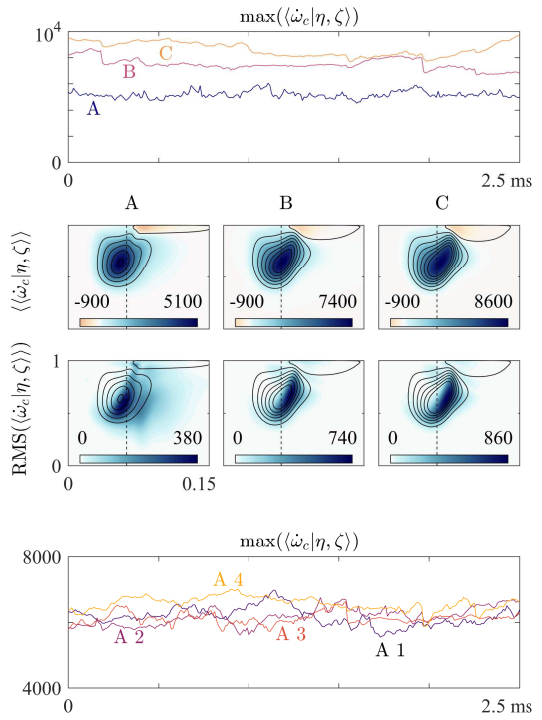

Figure 15: Temporal evolution of $\left\langle\dot{\omega}_{c} \mid \eta, \zeta\right\rangle$ for locations A, B and C (top). For locations A, $\mathrm{B}$ and $\mathrm{C}$ time averages of the conditional reaction rate $\left\langle\left\langle\dot{\omega}_{c} \mid \eta, \zeta\right\rangle\right\rangle$ and the time-based RMS are shown (middle). Note that the RMS is overlaid with contour lines of the temporal mean. In comparison, the evolution for the locations A1, A2, A3 and A4 with the same radial and axial position as $\mathrm{A}$, but different azimuthal locations, $0^{\circ}, 51^{\circ}, 103^{\circ}$ and $154^{\circ}$ respectively, are shown (bottom). The dashed vertical line marks $\xi_{\text {st }}$. 